\title{
Adaptive dynamics with interaction structure
}

Ben Allen

Martin A. Nowak

Ulf Dieckmann (dieckmann@iiasa.ac.at)

\section{Approved by}

Pavel Kabat

Director General and Chief Executive Officer

February 2015 Institute, its National Member Organizations, or other organizations supporting the work. 


\section{Adaptive dynamics with interaction structure}

Ben Allen ${ }^{1,2, *}$, Martin A. Nowak ${ }^{1,3,{ }^{* *}}$, and Ulf Dieckmann ${ }^{4, * * *}$

${ }^{1}$ Program for Evolutionary Dynamics, Harvard University, One Brattle Square, Cambridge, MA, 02138

${ }^{2}$ Department of Mathematics, Emmanuel College, 400 The Fenway, Boston, MA, 02115

${ }^{3}$ Department of Mathematics, Department of Organismic and Evolutionary Biology, Harvard University, One Brattle Square, Cambridge, MA, 02138

${ }^{4}$ Evolution and Ecology Program, International Institute for Applied Systems Analysis, Schlossplatz 1, A-2361 Laxenburg, Austria

*Corresponding author, Email: benjcallen@gmail.com

${ }^{* *}$ Email: nowak@fas.havard.edu

${ }^{* * *}$ Email: dieckmann@iiasa.ac.at 


\begin{abstract}
Evolutionary dynamics depend critically on a population's interaction structure - the pattern of which individuals interact with which others, depending on the state of the population and the environment. Previous research has shown, for example, that cooperative behaviors disfavored in well-mixed populations can be favored when interactions occur only between spatial neighbors or group members. Combining the adaptive dynamics approach with recent advances in evolutionary game theory, we here introduce a general mathematical framework for analyzing the long-term evolution of continuous game strategies for a broad class of evolutionary models, encompassing many varieties of interaction structure. Our main result, the "canonical equation of adaptive dynamics with interaction structure", characterizes expected evolutionary trajectories resulting from any such model, thereby generalizing a central tool of adaptive dynamics theory. Interestingly, the effects of different interaction structures and update rules on evolutionary trajectories are fully captured by just two real numbers associated with each model, which are independent of the considered game. The first, a structure coefficient, quantifies the effects on selection pressures, and thus on the shapes of expected evolutionary trajectories. The second, an effective population size, quantifies the effects on selection responses, and thus on the expected rates of adaptation. Applying our results to two social dilemmas, we show how the range of evolutionarily stable cooperative behaviors systematically varies with a model's structure coefficient.
\end{abstract}




\section{Introduction}

A key challenge for evolutionary biology is to understand how the mechanistic features of an evolutionary process affect the ultimate outcome of evolution. This is already quite challenging under the classical assumption of a well-mixed population (e.g., Dieckmann and Law, 1996; Champagnat et al., 2006). Recent decades, however, have seen a surge of interest in evolutionary processes in which ecological events are localized in some sense. Well-studied examples include evolution in spatially structured populations (Wright, 1943; Kimura and Weiss, 1964; Barton and Slatkin, 1986; Durrett and Levin, 1994; Tilman and Kareiva, 1997; Dieckmann et al., 2000; Rousset, 2004; Lieberman et al., 2005; Lion and van Baalen, 2008) in group- or demestructured populations (Wright, 1931; Wilson, 1977; Taylor, 1992; Hanski and Gilpin, 1997; Traulsen and Nowak, 2006; Ohtsuki, 2010), and in populations with active assortment by kin (Hamilton, 1971; Eshel and Cavalli-Sforza, 1982) or phenotype (Antal et al., 2009). These forms of localized interaction have significant consequences for the evolution of cooperation (Nowak and May, 1992; Killingback and Doebeli, 1996; Nakamaru et al., 1997; Mitteldorf and Wilson, 2000; Santos and Pacheco, 2005; Ohtsuki et al., 2006; Traulsen and Nowak, 2006; Taylor et al., 2007a; Fletcher and Doebeli, 2009; Helbing and Yu, 2009; van Veelen et al., 2012), host-parasite interactions (Hassell et al., 1991; Herre, 1993; Boots and Sasaki, 1999; Haraguchi and Sasaki, 2000; van Baalen, 2002; Read and Keeling, 2003; Boots et al., 2004), signaling (Krakauer and Pagel, 1995; Werfel and Bar-Yam, 2004), and life-history traits (Hanski and Gaggiotti, 2004).

In the context of evolutionary game theory, notions of locality are reflected in a model's interaction structure, which designates which individuals participate in game interaction with which others, depending on the current state of the population and the environment. Notions of locality may also be reflected in a model's update rule (Ohtsuki et al., 2006), which specifies how the outcomes of game interaction affect births, deaths, and other changes to the population and the environment.

It is thus of great interest (e.g. Nowak et al., 2010a) to understand how differences in the interaction structure and update rule affect the evolution of game behavior. Historically, this question has been investigated one model at a time, revealing both common themes and intriguing differences. In the case of discrete games, Tarnita et al. $(2009 b, 2011)$ have proposed a more 
general approach that exploits the Markov chain structure of evolutionary game models. However, no corresponding theory yet exists for continuous games (Brown and Vincent, 1987; McGill and Brown, 2007), in which payoffs depend continuously on quantitative trait values.

Here we provide a general mathematical framework for determining the evolutionary trajectories of continuous game strategies under different interaction structures and update rules. Our work merges adaptive dynamics theory (Nowak and Sigmund, 1990; Hofbauer and Sigmund, 1990; Dieckmann and Law, 1996; Metz et al., 1996; Geritz et al., 1997) - a framework for studying quantitative trait evolution when mutations are incremental and rare - with the approach of Tarnita et al. $(2009 b, 2011)$.

Our main result - which we term the "canonical equation of adaptive dynamics with interaction structure," characterizes expected evolutionary trajectories of strategies for any continuous game, interaction structure, and update rule satisfying basic assumptions. This result generalizes the canonical equation of adaptive dynamics (which describes expected trajectories of adaptive evolution in large well-mixed populations; Dieckmann and Law, 1996) to a broad class of models, including established models of evolution in groups (Wright, 1931; Taylor, 1992; Traulsen and Nowak, 2006; Ohtsuki, 2010), in space (Lieberman et al., 2005; Ohtsuki et al., 2006; Taylor et al., $2007 a$; Ohtsuki et al., 2007), with tag-based assortment (Antal et al., 2009), and in finite well-mixed populations with different forms of generational structure (Nowak et al., 2004; Imhof and Nowak, 2006; Lessard and Ladret, 2007; Traulsen et al., 2007).

Interestingly, we find that the effects of different interaction structures and update rules on the dynamics of adaptive evolution are fully captured by just two real numbers. First, a structure coefficient (Tarnita et al., 2009b) quantifies the effects on selection pressures, and thus on the shapes of expected evolutionary trajectories and on the strategies expected as evolutionary outcomes. Second, an effective population size quantifies the effects on selection responses and thus on the expected rates of adaptation. Importantly, these two numbers are independent of the considered evolutionary game. Varying the structure coefficient and effective population size in our equation thus enables rapid, comprehensive analysis of game-strategy trajectories for a wide variety of interaction structures and update rules, representing many different ecological contexts.

Below, we begin with a review and synthesis of relevant background and previous work on adaptive dynamics theory and evolutionary game theory. 
We then state the mathematical assumptions defining the class of models to which our results apply, which are further detailed in Appendix A. The canonical equation of adaptive dynamics with interaction structure is then presented, discussed, and applied to the analysis of two social dilemmas. Its derivation is detailed in Appendix B. We conclude with an exploration of connections between our work and other approaches, and highlight directions for future research.

\section{Background and synthesis of previous work}

\section{Adaptive dynamics theory}

Adaptive dynamics theory (Nowak and Sigmund, 1990; Hofbauer and Sigmund, 1990; Dieckmann and Law, 1996; Metz et al., 1996; Geritz et al., 1997; Dercole and Rinaldi, 2008) studies the long-term evolution of one or more quantitative traits, allowing the effects of complex interactions among individuals, including those implying frequency-dependent selection, to be analyzed. These interactions unfold on a short interaction timescale. Mutations are assumed to be rare, so that the evolving population is typically monomorphic, and its evolution can be studied on two separated timescales: the intermediate demographic timescale, on which competition between phenotypes results in fixation of one of them, and the longer evolutionary timescale, on which adaptation occurs via a sequence of trait-substitution events. Mutations are also assumed to be incremental, so that mutant phenotypes can be viewed as perturbations of resident phenotypes. Trait-substitution events start with a mutation and generally conclude with the fixation of either the mutant type or the resident type (Geritz et al., 2002; Geritz, 2005).

The canonical equation of adaptive dynamics (Dieckmann, 1994; Dieckmann et al., 1995; Dieckmann and Law, 1996) describes the expected dynamics, on the evolutionary timescale, of trait values in a large well-mixed population, as these values are updated through the successive fixation of new mutant types. In the case of a single real-valued trait $x$ evolving under birth-death dynamics with mutation, the canonical equation can be written as

$$
\frac{d x}{d t}=\left.\frac{1}{2} N \mu v \frac{\partial f\left(x^{\prime} ; x\right)}{\partial x^{\prime}}\right|_{x^{\prime}=x},
$$

where $t$ is evolutionary time, $N$ is the equilibrium population size, $\mu$ is the 
mutation probability per birth, $v$ is the variance in mutational step size, and $f\left(x^{\prime} ; x\right)$ is the invasion fitness of the mutant type $x^{\prime}$, given that the resident type is $x$. Invasion fitness is defined as the exponential growth rate of the mutants when they are rare (Metz et al., 1992). The right-hand side of eq. (1) equals the expected change in $x$ per unit time, averaged over all possible traitsubstitution events. The canonical equation, in setting the expected change equal to the actual change $d x / d t$, characterizes the expected dynamics of the trait value through evolutionary time (Dieckmann, 1994), as well as the deterministic dynamics under appropriate scaling limits (Champagnat et al., 2002).

The occurrence of the invasion fitness function $f\left(x^{\prime} ; x\right)$ in the canonical equation originates from the fact that, in a large well-mixed population, the probability of mutant fixation is proportional to $f\left(x^{\prime} ; x\right)$ when $f\left(x^{\prime} ; x\right)>0$, and is zero otherwise (Dieckmann, 1994; Dieckmann and Law, 1996; Dercole and Rinaldi, 2008; Durinx et al., 2008). In populations that are not large or not well-mixed, this proportionality may not hold (see Discussion). In such situations, we must fall back on a more general formulation of adaptive dynamics, by using fixation probabilities directly (Proulx and Day, 2002; Rousset, 2004; Imhof and Nowak, 2010), with expected evolutionary trajectories then being described by

$$
\frac{d x}{d t}=\left.N u(x) v \frac{\partial \rho\left(x^{\prime} ; x\right)}{\partial x^{\prime}}\right|_{x^{\prime}=x} .
$$

Here $u(x)$ denotes the per capita rate of mutant appearance in a monomorphic population of type $x$, and $\rho\left(x^{\prime} ; x\right)$ denotes the fixation probability of a mutant of type $x^{\prime}$ in such a population - that is, the probability that a new mutant type $x^{\prime}$, starting from a single individual, will eventually displace a resident population of type $x$. For large well-mixed populations, and with $b(x)$ denoting the per capita birth rate of type $x$, eqs. (1) and (2) are connected by the relations $u(x)=\mu b(x)$ and $\rho\left(x^{\prime} ; x\right)=\max \left\{0, f\left(x^{\prime} ; x\right) / b(x)\right\}$ (Dieckmann and Law, 1996).

Equation (2) illustrates the separation of timescales inherent in the adaptive dynamics approach. The whole of eq. (2) describes trait dynamics on the evolutionary timescale. These dynamics depend, in turn, on the outcome of resident-mutant competition on the demographic timescale, as summarized by the fixation probability $\rho\left(x^{\prime} ; x\right)$. To apply eq. (2), one must obtain $\rho\left(x^{\prime} ; x\right)$ (or, at minimum, its first-order behavior near $x^{\prime}=x$ ) from analysis of resident-mutant competition under the evolutionary model in question. 
This may be a difficult task, depending on the complexity of the model. One contribution of the present work is to obtain expressions for this first-order behavior of $\rho\left(x^{\prime} ; x\right)$ for a general class of models in evolutionary game theory.

\section{Individual-based evolutionary game (IBEG) models}

Evolutionary game theory (Maynard Smith and Price, 1973; Taylor and Jonker, 1978; Weibull, 1997; Cressman, 1992; Hofbauer and Sigmund, 1998, 2003; Nowak and Sigmund, 2004) is a powerful approach to studying the evolution of social behavior. A game represents a particular mode of interaction, the outcome of which is summarized in payoffs assigned to each participant. Evolving game strategies may be either discrete or continuous. For continuous games (Brown and Vincent, 1987; McGill and Brown, 2007), the strategies are real numbers or vectors representing quantitative traits, and the payoffs to participants are continuous functions of these strategies.

While early formulations of evolutionary game theory focused on notions of evolutionary stability (Maynard Smith and Price, 1973) and on frequency dynamics in infinite populations (Taylor and Jonker, 1978), much recent attention has been devoted to individual-based evolutionary game modelswhich we abbreviate as "IBEG models" - in which births, deaths, game interactions, and other events are represented explicitly (Nowak and May, 1992; Durrett and Levin, 1994; Killingback and Doebeli, 1996; Nakamaru et al., 1997; van Baalen and Rand, 1998; Mitteldorf and Wilson, 2000; Nowak et al., 2004; Santos and Pacheco, 2005; Ohtsuki et al., 2006; Traulsen and Nowak, 2006; Szabó and Fáth, 2007; Taylor et al., 2007 ; Antal et al., 2009; Tarnita et al., 2009a; Nowak et al., 2010a; Perc and Szolnoki, 2010; van Veelen et al., 2012). In contrast to traditional evolutionary game theory, IBEG models allow investigation of how localized interaction, population size, and other factors affect evolutionary game competition.

Here we provide a synthesis and general vocabulary for IBEG models, highlighting several critical features. We describe these models in biological terms, noting, however, that many IBEG models are applicable to cultural evolution through the spreading of behaviors or ideas. We also note that, while the term "individual-based" often carries the connotation of models analyzed through numerical analysis and computer simulation, here we use it to characterize the level at which the considered evolutionary game dynamics are defined, irrespective of the method subsequently applied for their analysis. IBEG models appearing in the literature generally have the mathemat- 
ical structure of a Markov chain. That is, they can be represented as a collection of states with transition probabilities between them. (Deterministic and/or continuous-state models can be understood as special and/or limiting cases of Markov chain models.) In this terminology, the "state" by definition provides a full snapshot of the evolutionary process, containing all information relevant to the determination of future states. At minimum, this includes the number and strategies of all living individuals. The inclusion of other information depends on the model in question. For example, in models of group-structured populations (Traulsen and Nowak, 2006; Tarnita et al., 2009a; Ohtsuki, 2010; Fu et al., 2012), the state includes the group affiliations of all individuals. In models with dynamic social networks (Pacheco et al., 2006a,b; Perc and Szolnoki, 2010; Wu et al., 2010; Fehl et al., 2011; Rand et al., 2011), the state includes the current network topology. Models have also been studied in which the state includes individuals' ages, developmental stages, memories of past events, and/or states of health, as well as the environmental conditions each of them experiences.

An IBEG model must incorporate a scheme for designating when game interactions occur, and between which pairs or sets of individuals. We call this scheme the model's interaction structure. Models of well-mixed populations (e.g., Nowak et al., 2004; Imhof and Nowak, 2006; Lessard and Ladret, 2007; Traulsen et al., 2007) typically employ a trivial interaction structure: at all time steps, each individual interacts equally with each of the other individuals. Other models use interaction structures that incorporate some notion of locality. For example, individuals may interact only if they are members of the same group (Taylor, 1992; Traulsen and Nowak, 2006; Ohtsuki, 2010), neighbors on a lattice (Nowak and May, 1992; Wilson et al., 1992; Durrett and Levin, 1994; Killingback and Doebeli, 1996; Nakamaru et al., 1997; Hauert and Doebeli, 2004; Helbing and Yu, 2009; Roca et al., 2009), or are connected through the (current) social network (van Baalen and Rand, 1998; Santos and Pacheco, 2005; Ohtsuki et al., 2006; Pacheco et al., 2006a,b; Taylor et al., 2007a; Perc and Szolnoki, 2010; Allen et al., 2012). In the most abstract sense, a model's interaction structure can be understood as a mapping from its current state to the current collection of game-interaction partners.

Once game interactions have taken place, births, deaths, movements, and other relevant events must be determined according to another scheme, depending (often stochastically) on payoffs. Following Ohtsuki et al. (2006), we call this scheme the model's update rule. For well-mixed population models, 
update rules usually have the property that each individual's expected offspring number is an increasing function of its own payoff (Nowak et al., 2004; Imhof and Nowak, 2006; Lessard and Ladret, 2007). For models with nontrivial interaction structure, there is no canonical choice of update rule, and different choices can lead to strikingly different outcomes. For example, Ohtsuki et al. (2006) explored three different update rules for evolution on graphs. For one ("Birth-Death"), the benefits of cooperator clustering are opposed by a spatial competition effect separate from game interaction, rendering cooperation ineffective. (A similar effect appears in the group-selection model of Taylor, 1992 and Ohtsuki, 2010.) For two other update rules ("Death-Birth" and "Imitation"), the spatial competition effect is weaker and cooperation can be favored. Thus the choice of update rule is a modeling decision that must be treated with appropriate care.

Finally, the probabilities of transition between states - and hence the dynamics and outcomes of evolutionary competition - depend on the game, the interaction structure, and the update rule of the considered IBEG model. We therefore view the game, interaction structure, and update rule as three key ingredients of any IBEG model, leaving the abstract formalization of this idea to future work. An important and attractive feature of IBEG models is that the considered game can be varied independently from the considered interaction structure and the considered update rule. Varying the latter two ingredients can elucidate, for example, how the success of a particular trait or behavior varies with the geometry of the considered environment.

\section{Structure coefficient}

Tarnita et al. (2009b; 2011; see also Nowak et al., 2010a) have recently pioneered a general approach to studying IBEG models on the demographic timescale. This approach focuses on the Markov chain structure of these models, abstracting from the details of births, deaths, and game interactions.

Tarnita et al. (2009b) considered IBEG models involving pairwise games with two strategies, described by $2 \times 2$ payoff matrices,

\begin{tabular}{c|cc} 
& $X$ & $Y$ \\
\hline$X$ & $a$ & $b$ \\
$Y$ & $c$ & $d$.
\end{tabular}

Above, $X$ and $Y$ are the two considered strategies, $a$ and $b$ are the payoffs received by $X$ when playing against $X$ and $Y$, respectively, and $c$ and $d$ are 
the payoffs received by $Y$ when playing against $X$ and $Y$, respectively. In the Markov chain representation of such an IBEG model, each transition probability between any pair of states can be considered a function of the payoff values $a, b, c$, and $d$, with this function depending in turn on the interaction structure and update rule of the considered model. Tarnita et al. (2009b) imposed basic differentiability and symmetry assumptions on the transition probabilities as functions of the payoff values. On this basis, they proved that any IBEG model satisfying their assumptions can be characterized by a realvalued "structure coefficient" $\sigma$. The value of $\sigma$ depends on the interaction structure and update rule, but is independent of the game. Given a particular game of the form (3), strategy $X$ is favored over strategy $Y$-in the sense that $X$ has greater fixation probability than $Y$ under weak selection-if and only if

$$
\sigma a+b>c+\sigma d .
$$

The utility of this result is that, once the value of $\sigma$ has been determined for a particular combination of interaction structure and update rule (see examples in Table 1), condition (4) can be used to determine which strategy is favored in any $2 \times 2$ game. In short, $\sigma$ quantifies how a particular combination of interaction structure and update rule affects the selection pressure on game strategies. For a well-mixed population, $\sigma$ approaches 1 as population size approaches infinity; thus, $\sigma=1$ can be interpreted as a baseline value. In this case, the same-type payoffs $a$ and $d$ and the oppositetype payoffs $b$ and $c$ are of equal importance in determining evolutionary success. As $\sigma$ is increased above 1 , the same-type payoffs become increasingly important. Conversely, as $\sigma$ is decreased below 1, the opposite-type payoffs become increasingly important. Accordingly, the former case favors cooperative strategies, whereas the latter case hinders them (Tarnita et al., $2009 b)$.

We caution that the structure coefficient does not merely quantify assortment of strategies or genetic relatedness between interaction partners. Its value also depends, for example, on any indirect competition effects that may be subsumed in the update rule. We will revisit the interpretation of $\sigma$ throughout this work. In particular, as part of our results, we derive an expression for $\sigma$ in terms of the dependence of fixation probabilities on game payoffs. 


\section{Mathematical framework}

Our main result, an equation describing the evolutionary trajectories of continuous game strategies, extends the approach of Tarnita et al. (2009b) from the demographic to the evolutionary timescale, using the adaptive dynamics framework. The resultant equation applies to any IBEG model satisfying a set of basic assumptions. In this section, we present these assumptions verbally, saving rigorous mathematical formulations for Appendix A. We separate our assumptions into those applying to the interaction timescale (labeled G1-G2), the demographic timescale (D1-D6), and the evolutionary timescale (E1-E3).

\section{Interaction timescale: Game strategies and payoffs}

Interactions are modeled as a continuous game with payoff function $A$. The possible strategies are represented as real numbers or vectors. In the twoplayer case, $A(x ; y)$ denotes the payoff to an individual of strategy $x$ interacting with an individual of strategy $y$. For $n$-player games, the payoff function is written $A\left(x ; y_{1}, \ldots, y_{n-1}\right)$. In this case, the first argument $x$ denotes the strategy of the focal individual receiving the payoff, and the remaining $n-1$ arguments are the strategies of the other game participants. We place the following assumptions on $A$ :

G1. $A$ is positive and twice differentiable in all of its arguments, at least in the case that all game participants use approximately the same strategy.

G2. For games with more than two players, the arrangement of interaction partners does not matter, i.e., the value of $A\left(x ; y_{1}, \ldots, y_{n-1}\right)$ does not change if the arguments $y_{1}, \ldots, y_{n-1}$ are permuted.

\section{Demographic timescale: Resident-mutant competition}

On the demographic timescale, we are interested in episodes of competition between a resident strategy $x$ and a mutant strategy $x^{\prime}$. Four payoff values arise in this competition, and we introduce the following shorthand notation for these:

$$
\begin{aligned}
a_{\mathrm{MM}}=A\left(x^{\prime} ; x^{\prime}\right) & a_{\mathrm{MR}}=A\left(x^{\prime} ; x\right) \\
a_{\mathrm{RM}}=A\left(x ; x^{\prime}\right) & a_{\mathrm{RR}}=A(x ; x) .
\end{aligned}
$$


Following the approach of Tarnita et al. (2009b), we focus on the Markov chain representing resident-mutant competition under the considered IBEG model, abstracting from lower-level biological details. This Markov chain is initiated in a state corresponding to the appearance of a single new mutant. Eventually, the Markov chain will reach a state corresponding to either mutant fixation or extinction. We denote by $\rho$ the probability that mutant fixation is reached.

We impose six assumptions on how the Markov chains transition probabilities vary with respect to $a_{\mathrm{MM}}, a_{\mathrm{MR}}, a_{\mathrm{RM}}$, and $a_{\mathrm{RR}}$. These assumptions implicitly restrict the choices of interaction structures and update rules in IBEG models that can be analyzed using our approach.

D1. The set of possible states is finite,

D2. The Markov chain's transition probabilities are completely determined by the payoff values $a_{\mathrm{MM}}, a_{\mathrm{MR}}, a_{\mathrm{RM}}$, and $a_{\mathrm{RR}}$,

D3. For any values of $a_{\mathrm{MM}}, a_{\mathrm{MR}}, a_{\mathrm{RM}}$, and $a_{\mathrm{RR}}$, mutant fixation is possible from any initial state corresponding to the appearance of a single new mutant,

D4. The Markov chain's transition probabilities vary twice differentiably with respect to $a_{\mathrm{MM}}, a_{\mathrm{MR}}, a_{\mathrm{RM}}$, and $a_{\mathrm{RR}}$,

D5. The fixation probability $\rho$ is unaffected if $a_{\mathrm{MM}}, a_{\mathrm{MR}}, a_{\mathrm{RM}}$, and $a_{\mathrm{RR}}$ are each multiplied by a positive constant $K>0$,

D6. The fixation probability $\rho$ is increasing in $a_{\mathrm{MM}}$ and $a_{\mathrm{MR}}$, and decreasing in $a_{\mathrm{RM}}$ and $a_{\mathrm{RR}}$.

Assumption D2 requires that the evolving strategies affect only the game payoffs; if the evolving strategies also affected the interaction structure or update rule, then the Markov chain's transition probabilities would not be completely determined by $a_{\mathrm{MM}}, a_{\mathrm{MR}}, a_{\mathrm{RM}}$, and $a_{\mathrm{RR}}$, but would depend on $x$ as well. Assumption D5 guarantees that the fixation probabilities are insensitive to changes in the units in which payoffs are measured.

While these assumptions apply to a wide variety of IBEG models (a sample of which are listed in Table 1), they exclude models with infinitely many states (e.g., those with infinite population size or continuous space) and models in which behaviors affecting the interaction structure (e.g., homophily) or update rule (e.g., migration) coevolve with the game strategies. 
The six assumptions above are compatible with those stipulated by Tarnita et al. (2009b). Consequently, there is a structure coefficient, satisfying (4), associated to each combination of interaction structure and update rule satisfying these assumptions.

\section{Evolutionary timescale: Mutation and trait substitu- tion}

Following the adaptive dynamics approach, we make the following two assumptions regarding the mutation process:

E1. Mutations are rare, so that the evolving population is typically monomorphic, and long-term evolution can be described as a sequence of trait substitutions.

E2. Mutation is directionally unbiased.

E3. Mutation is incremental, so that the mutant strategy $x^{\prime}$ is always close to the resident strategy $x$.

\section{Main result: Canonical equation of adaptive dynamics with interaction structure}

This section presents our central result, the canonical equation of adaptive dynamics with interaction structure. This equation describes expected evolutionary trajectories of a game strategy $x$ for any combination of continuous game (satisfying Assumptions G1-G2), update rule and interaction structure (satisfying Assumptions D1-D6), and mutation process (satisfying Assumptions E1-E3).

\section{Univariate traits and pairwise games}

We begin with the simple case of a pairwise (two-player) game $A(x ; y)$, in which the strategies $x$ and $y$ are univariate (real numbers). The canonical equation of adaptive dynamics with interaction structure in this case is

$$
\frac{d x}{d t}=N_{\mathrm{e}} \frac{N-1}{N} \frac{u(x) v}{A(x ; x)}\left(\left.\frac{\partial A\left(x^{\prime} ; x\right)}{\partial x^{\prime}}\right|_{x^{\prime}=x}+\left.\frac{\sigma-1}{\sigma+1} \frac{\partial A\left(x ; x^{\prime}\right)}{\partial x^{\prime}}\right|_{x^{\prime}=x}\right),
$$


where $\sigma$ is the structure coefficient and $N_{\mathrm{e}}$ is the effective population size (defined below). As in eq. (2), $t$ is evolutionary time, $N$ is the equilibrium population size, $u(x)$ is the per capita rate of mutant appearance, and $v$ is the variance in mutational step size.

This result is obtained by first showing that expected evolutionary trajectories are related to fixation probabilities as in eq. (2), and then showing that the relevant derivatives of fixation probabilities can be expressed in terms of the structure coefficient and effective population size. The full derivation is given in Appendix B. We now discuss several aspects of this result in detail.

\section{Structure coefficient and shapes of evolutionary trajec- tories}

First we note that all effects of the interaction structure and update rule on the shape of evolutionary trajectories - in particular on the strategies corresponding to evolutionary equilibria - are described by the proportionality relation

$$
\left.\frac{d x}{d t} \propto \frac{\partial A\left(x^{\prime} ; x\right)}{\partial x^{\prime}}\right|_{x^{\prime}=x}+\left.\frac{\sigma-1}{\sigma+1} \frac{\partial A\left(x ; x^{\prime}\right)}{\partial x^{\prime}}\right|_{x^{\prime}=x},
$$

where the symbol $\propto$ means "proportional to". The two terms of eq. (6) express a mutation's evolutionary success in terms of its effect on the payoff of its bearer (first term), as well as its effect on the payoffs of those with whom the bearer interacts (second term). In the limit of a large, well-mixed population $(\sigma \rightarrow 1)$, only the bearer's payoff matters. But with nontrivial interaction structure and/or update rule $(\sigma \neq 1)$, a mutant's effect on the payoffs of others is relevant, with the sign of $\sigma-1$ identifying whether helpful or harmful effects are favored by the second term.

We also derive in Appendix B an expression for $\sigma$ in terms of the behavior of the mutant fixation probability $\rho$ with respect to the game payoffs:

$$
\sigma=\left(\frac{\partial \rho}{\partial a_{\mathrm{MM}}}-\frac{\partial \rho}{\partial a_{\mathrm{RR}}}\right) /\left(\frac{\partial \rho}{\partial a_{\mathrm{MR}}}-\frac{\partial \rho}{\partial a_{\mathrm{RM}}}\right),
$$

with all derivatives taken at $a_{\mathrm{MM}}=a_{\mathrm{MR}}=a_{\mathrm{RM}}=a_{\mathrm{RR}}=1$. We note in passing that this expression also applies to the result of Tarnita et al. (2009b), with $a, b, c$, and $d$ in place of $a_{\mathrm{MM}}, a_{\mathrm{MR}}, a_{\mathrm{RM}}$, and $a_{\mathrm{RR}}$, respectively. We additionally show in Appendix B that $\sigma$ is always positive, as a consequence of Assumption D6. 
The appearance of the ratio $(\sigma-1) /(\sigma+1)$ in eq. (6) can be understood by the following argument. By condition (4), a mutant strategy $x^{\prime}$ is favored over the resident strategy $x$ if and only if

$$
\sigma A\left(x^{\prime} ; x^{\prime}\right)+A\left(x^{\prime} ; x\right)-A\left(x ; x^{\prime}\right)-\sigma A(x ; x)>0 .
$$

For $x^{\prime}=x$, the quantity on the left-hand side vanishes. Therefore, to first order in $x^{\prime}-x$, mutant favorability is determined by the sign of

$$
\begin{array}{r}
\sigma\left(\left.\frac{\partial A\left(x^{\prime} ; x\right)}{\partial x^{\prime}}\right|_{x^{\prime}=x}+\left.\frac{\partial A\left(x ; x^{\prime}\right)}{\partial x^{\prime}}\right|_{x^{\prime}=x}\right)+\left.\frac{\partial A\left(x^{\prime} ; x\right)}{\partial x^{\prime}}\right|_{x^{\prime}=x}-\left.\frac{\partial A\left(x ; x^{\prime}\right)}{\partial x^{\prime}}\right|_{x^{\prime}=x} \\
=\left.(\sigma+1) \frac{\partial A\left(x^{\prime} ; x\right)}{\partial x^{\prime}}\right|_{x^{\prime}=x}+\left.(\sigma-1) \frac{\partial A\left(x ; x^{\prime}\right)}{\partial x^{\prime}}\right|_{x^{\prime}=x}
\end{array}
$$

Dividing by $\sigma+1$, we obtain the coefficients in eq. (6).

Values of $\sigma$ for a variety of IBEG models are shown in Table 1.

\section{Effective population size and speed along evolutionary trajectories}

We next observe that the rate of evolution in eq. (5) is proportional to the effective population size $N_{\mathrm{e}}$. The effective population size is defined here in terms of the selection response, i.e. through the likelihood of beneficial mutations to fixate. Our definition is motivated by Kimura's (1964) result that, for a class of models amenable to one-dimensional diffusion approximation, a beneficial mutation of selective advantage $s$ has fixation probability

$$
\rho \approx \frac{1-e^{-2 s N_{\mathrm{e}} / N}}{1-e^{-2 s N_{\mathrm{e}}}}
$$

Differentiating with respect to $s$ and solving for $N_{\mathrm{e}}$ yields

$$
N_{\mathrm{e}}=\left.\frac{N^{2}}{N-1} \frac{\partial \rho}{\partial s}\right|_{s=0}
$$

which we take as our definition of $N_{\mathrm{e}}$. We note that in this work the actual and effective population sizes, $N$ and $N_{\mathrm{e}}$, respectively, pertain to the demographic equilibrium of the resident population, rather than to its current state at a given time. 
Our $N_{\mathrm{e}}$ agrees with the variance effective population size (Kimura, 1964) and the eigenvalue effective population size (Ewens, 1982; see also Rousset, 2004) for models for which these are defined. Our definition is similar in spirit to the adaptation effective population size defined by Campos and Wahl (2009). More generally, it is proven for some models and conjectured more generally (Metz, 2011) that the notion of effective population size as a quantity scaling the rate of adaptation - as in eq. (6) - is equivalent to the more familiar notion of effective population size as a quantity inversely proportional to the strength of neutral drift.

A convention implicit in eq. (8) is that the Wright-Fisher (non-overlapping generations) model is taken as a baseline. Consequently, $N_{\mathrm{e}}=N$ for the Wright-Fisher model, while $N_{\mathrm{e}}$ is greater than (less than) $N$ for population structures that promote (inhibit) the fixation of beneficial mutations, relative to this model. Interestingly, many overlapping-generation IBEG models (Moran, 1958; Dieckmann, 1994; Nowak et al., 2004) have $N_{\mathrm{e}}=N / 2$. Values of $N_{\mathrm{e}}$ for a variety of models are shown in Table 1.

\section{Rates of mutant appearance}

In the canonical equation (5), the process by which new mutations appear is deliberately left unspecified. This flexibility allows for the application of eq. (5) to a wide variety of biological and cultural evolutionary processes.

For biological evolution, it is natural to set $u(x)$ equal to $\mu b(x)$, where $b(x)$ is the birth rate in a monomorphic population of strategy $x$, and $\mu$ is the mutation probability per birth as in eq. (1). An important special case occurs when payoff is equal to birth rate in monomorphic populations, so that $b(x)=A(x ; x)$. In this case the ratio $u(x) / A(x ; x)$ in eq. (5) simplifies to $\mu$.

For cultural evolution, $u(x)$ can be interpreted as the per capita rate at which new ideas or behaviors are introduced. Depending on the details of the considered evolutionary process, it may then be reasonable to assume $u(x)$ to be constant (if innovation is unrelated to strategies or payoffs), proportional to $A(x ; x)$ (if high payoffs induce greater levels of innovation), or inversely proportional to $A(x ; x)$ (if innovation is induced by discontent with one's current payoff). 


\section{Recovery of original canonical equation}

Dieckmann (1994) derived the original canonical equation of adaptive dynamics, eq. (1), using a continuous-time birth-death model in a large wellmixed population. For this model, $\sigma=1$ and $N_{\mathrm{e}}=N / 2$. If we equate an individual's birth rate with the average payoff obtained from all other individuals and assume a constant death rate, then $u(x)=\mu A(x ; x), f\left(x^{\prime} ; x\right)=$ $A\left(x^{\prime} ; x\right)-1$, and eq. (5) reduces to the original canonical equation eq. (1).

\section{Multivariate traits}

For continuous games with multivariate ( $d$-dimensional) strategies, $x=\left(x_{1}, \ldots, x_{d}\right)$, evolutionary trajectories are described by the system of equations

$$
\frac{d x_{i}}{d t}=N_{\mathrm{e}} \frac{N-1}{N} \frac{u(x) v_{i}}{A(x ; x)}\left(\left.\frac{\partial A\left(x^{\prime} ; x\right)}{\partial x_{i}^{\prime}}\right|_{x^{\prime}=x}+\left.\frac{\sigma-1}{\sigma+1} \frac{\partial A\left(x ; x^{\prime}\right)}{\partial x_{i}^{\prime}}\right|_{x^{\prime}=x}\right),
$$

for $i=1, \ldots, d$. Here, the mutational step size variance $v_{i}$ is given the subscript $i$ to reflect that mutations in different components $i$ of $x$ may be differently distributed.

Equation (10) applies when mutations in each component of $x$ are independent. Correlations among mutations in different components can be readily accommodated, following a formalism described by Dieckmann and Law $(1996, \S 6.2)$.

\section{Multilateral interactions}

Our result extends to games involving any number of players. We show in Appendix $\mathrm{C}$ that an $n$-player game $B\left(x ; y_{1}, \ldots, y_{n}\right)$ can be replaced by the pairwise game with payoff function $A(x ; y)=B(x ; y, \ldots, y)$-more specifically, the payoff value $B\left(x ; y_{1}, \ldots, y_{n-1}\right)$ can be replaced by the arithmetic mean of $A\left(x ; y_{j}\right)$ for $j=1, \ldots, n-1$. In other words, one can suppose that the pairwise game $A$ is played with each of the $n-1$ partners and the results are then averaged; this substitution leaves evolutionary trajectories unchanged.

This surprising simplification arises from the differentiability and symmetry properties, G1 and G2, imposed on the game. It does not imply that the set of interaction partners is typically monomorphic. 


\section{Range of applications}

The power of our result lies in its generality. To obtain the expected evolutionary trajectory of a particular continuous strategy under a particular IBEG model, one need only substitute the appropriate payoff function $A$ and values of $\sigma$ and $N_{\mathrm{e}}$ into the canonical equation with interaction structure, eq. (10). This saves the labor of analyzing each combination of game, interaction structure, and update rule from scratch. Moreover, our result enables comparisons both within classes of models (e.g., graphs with different topologies) and across such classes (e.g., models with pre-defined groups versus those with tag-based assortment). Allowing $\sigma$ and $N_{\mathrm{e}}$ to vary across all positive values reveals, all at once, the range of potential trajectories a quantitative trait can follow under all conceivable ecological scenarios fulfilling the rather general assumptions above.

\section{Examples: Social dilemmas}

We now illustrate the power of our framework by considering two social dilemmas with continuous strategies. In each case we find that, as the structure coefficient increases, new cooperative equilibria arise and existing equilibria shift toward greater levels of cooperation.

\section{Example 1: One-shot continuous Prisoner's Dilemma}

We first investigate the continuous Prisoner's Dilemma (Killingback et al., 1999; Wahl and Nowak, 1999b, a; Killingback and Doebeli, 2002). This models a pairwise social dilemma with a continuous range of cooperativity levels, quantified by a nonnegative real number $x$. In each interaction, a player with cooperativity $x$ pays a cost $C(x)$ to produce a benefit $B(x)$ for the other player. We assume that the functions $C(x)$ and $B(x)$ are twice differentiable, strictly increasing, and satisfy $C(0)=B(0)=0$ (so that $x=0$ corresponds to no cooperation) and $C(x)<B(x)$ for $x>0$. The payoff function thus is

$$
A(x, y)=-C(x)+B(y) .
$$

Applying eq. (6), we immediately obtain the cooperativity dynamics un- 
der any IBEG model satisfying our assumptions:

$$
\frac{d x}{d t} \propto-C^{\prime}(x)+\frac{\sigma-1}{\sigma+1} B^{\prime}(x) .
$$

For large well-mixed populations $(\sigma=1)$, these dynamics depend only on the marginal cost $C^{\prime}(x)$ of cooperation. The second term contributes when $\sigma \neq 1$ and captures how a mutant's success depends on the marginal benefit $B^{\prime}(x)$ it gives to others. We see that, depending on the interaction structure and update rule, a mutant's success can increase $(\sigma>1)$ or decrease $(\sigma<1)$ with this marginal benefit. The ratio $(\sigma-1) /(\sigma+1)$ can therefore be interpreted as quantifying the "potential for altruism" (Gardner, 2010) afforded by a particular combination of interaction structure and update rule.

Cooperativity increases from its current value $x$ if and only if both $\sigma>1$ and

$$
\frac{B^{\prime}(x)}{C^{\prime}(x)}>\frac{\sigma+1}{\sigma-1}
$$

Conversely, if $\sigma \leq 1$ or if $B^{\prime}(0) / C^{\prime}(0)<\frac{\sigma+1}{\sigma-1}$, cooperativity will not increase from an initial value of zero.

The left-hand side of condition (12) is the marginal-benefit-to-marginalcost ratio of an increase in cooperativity. Condition (12) thus is a differential version of the benefit-to-cost thresholds, such as Hamilton's rule, that frequently arise as conditions for the evolution of cooperation (reviewed by Nowak, 2006).

Cooperativity equilibria occur at values $x=x^{*}$ satisfying $B^{\prime}\left(x^{*}\right) / C^{\prime}\left(x^{*}\right)=$ $(\sigma+1)(\sigma-1)$. An equilibrium $x^{*}$ is stable if

$$
\left.\frac{d}{d x} \frac{B^{\prime}(x)}{C^{\prime}(x)}\right|_{x=x^{*}}<0
$$

that is, if the marginal-benefit-to-marginal-cost ratio of cooperation is decreasing at $x=x^{*}$. Conversely, unstable equilibria occur when this ratio is increasing. In the latter case, $x^{*}$ can be understood as a cooperativity threshold, in that cooperativity dwindles when $x<x^{*}$ but becomes self-reinforcing when $x>x^{*}$.

We can neatly summarize these results in a bifurcation diagram (Figure 1 ), in which the structure coefficient serves as the control parameter. This diagram shows how greater values of $\sigma$ yield higher levels of evolutionarily stable cooperativity. In general, our main result enables constructing such a bifurcation diagram for any continuous game, showing how the evolutionary trajectories following eq. (10) vary with a model's structure coefficient. 


\section{Example 2: Iterated Prisoner's Dilemma with stochas- tic reactive strategies}

The iterated Prisoner's Dilemma with stochastic reactive strategies (Nowak and Sigmund, 1990, 1992, 1993; Imhof and Nowak, 2010) is an elegant and well-studied example of multivariate adaptive dynamics. Here we consider the Prisoner's Dilemma game

\begin{tabular}{c|cc} 
& $C$ & $D$ \\
\hline$C$ & $b-c$ & $-c$ \\
$D$ & $b$ & 0
\end{tabular}

played an infinite number of times between two players. Each player's move depends stochastically on the previous move of the other player. A strategy is characterized by a pair of probabilities, $(p, q) \in[0,1] \times[0,1]$. The strategy component $p$ (resp., $q$ ) represents the probability of cooperating in response to cooperation (resp., defection) by the other player. Thus, the strategy $(0,0)$ corresponds to "always defect" (ALLD), $(1,1)$ to "always cooperate" (ALLC), and $(1,0)$ to "tit-for-tat" (TFT). The payoff to each player is averaged over an infinite number of iterations; hence the choice of opening move is unimportant.

For this game, the payoff to a player with strategy $\left(p_{1}, q_{1}\right)$ interacting with a player with strategy $\left(p_{2}, q_{2}\right)$, as calculated by Nowak and Sigmund (1990), is

$$
A\left(p_{1}, q_{1} ; p_{2}, q_{2}\right)=\frac{-c\left(q_{1}+p_{1} q_{2}-q_{1} q_{2}\right)+b\left(q_{2}+p_{2} q_{1}-q_{2} q_{1}\right)}{1-\left(p_{1}-q_{1}\right)\left(p_{2}-q_{2}\right)} .
$$

We study the adaptive dynamics of the cooperation probabilities $p$ and $q$ using the multivariate canonical equation with interaction structure eq. (10). For simplicity, we assume that mutations in $p$ and $q$ are independent, and of equal expected magnitude, but this assumption can readily be relaxed.

Upon computing the four partial derivatives of $A$ as required by eq. (10), it follows immediately that, for any IBEG model satisfying our assumptions, the expected evolutionary trajectories trace concentric circles around the point $(p, q)=(1,0)$ corresponding to TFT. It further follows that, along these trajectories, cooperation increases $(d p / d t>0$ and $d q / d t>0)$ if

$$
(\sigma+1)(-c+b(p-q))+(\sigma-1)(-c(p-q)+b)>0
$$


and decreases $(d p / d t<0$ and $d q / d t<0)$ otherwise.

The observations above fully determine the evolutionary trajectories for this game under any interaction structure and update rule. Figure 2 illustrates these results, showing how the range of stable equilibria with positive $p$ and $q$ values increases with the structure coefficient $\sigma$.

\section{Discussion}

\section{Summary}

Although patterns of interaction and replacement can have complex and varied effects on a population's evolution, our results here show that, for the purposes of determining the expected trajectories of continuous strategies under a broad class of evolutionary game models, these effects are fully quantified by just two real numbers, the effective population size and the structure coefficient. This remarkable simplification arises from the assumptions of rare and incremental mutation that underlie the adaptive dynamics framework.

Our results facilitate a broader approach to the study of continuous strategy evolution. Rather than studying individual combinations of a game, an interaction structure, and an update rule, one can derive the values of the structure coefficient and effective population size for a particular choice of interaction structure and update rule (as in the examples shown in Table 1 ), and thereby immediately understand their effects on adaptive dynamics for any continuous game. Or, as in Figures 1 and 2, one can focus on a single game and, by varying $\sigma$ and $N_{\mathrm{e}}$, understand how adaptive dynamics and outcomes for this game vary under different interaction structures and update rules.

Our work also unifies previous findings that spatial structure can promote cooperation in continuous-strategy social dilemmas (van Baalen and Rand, 1998; Killingback et al., 1999; Le Galliard et al., 2003, 2005; Ifti et al., 2004). Our work shows that, across models and games, the strength of this spatial benefit to cooperation is quantified by the structure coefficient $\sigma$. Indeed, whenever $\sigma>1$, the coefficient of the second term of eq. (10) is positive, indicating that selection favors strategies that increase the payoffs of others. The two examples in the preceding section show how both the range and the intensity of evolutionarily stable cooperative behaviors can increase with $\sigma$. 


\section{Fixation probability versus invasion fitness}

We depart from most prior work on adaptive dynamics in structured populations (van Baalen and Rand, 1998; Ferrière and Le Galliard, 2001; Metz and Gyllenberg, 2001; Durinx et al., 2008) in our use of fixation probabilities in place of invasion fitness - defined as the exponential growth rate of a rare mutant subject to the current environment (Metz et al., 1992; Rand et al., 1994; Dieckmann and Law, 1996; Metz et al., 1996; Heino et al., 1998).

This concept is natural in well-mixed populations. Additionally, for some models with nontrivial interaction structure, it can be shown that assortment patterns are likely to equilibrate (in some sense) while a mutant is still rare (Matsuda et al., 1992; van Baalen and Rand, 1998; Ferrière and Le Galliard, 2001; Ohtsuki and Nowak, 2008). The invasion fitness can then be derived assuming that such equilibration has occurred. However, such an approach does not necessarily account for how the mutant's fixation probability is affected by its dynamics prior to this equilibration. It is also unclear whether the separation of timescales needed for defining an exponential phase can be expected to hold for all models of resident-mutant competition with interaction structure. Basing adaptive dynamics directly on fixation probabilities (Proulx and Day, 2002; Rousset, 2004; Imhof and Nowak, 2010) eschews such difficulties.

The direct use of fixation probabilities is therefore attractive when these can be determined in addition to, or independently of, invasion fitness. By contrast, where fixation probabilities need to be inferred from invasion fitness, they provide no marginal benefit. Moreover, fixation probabilities are less appropriate for understanding processes of evolutionary branching (Metz et al., 1992; Geritz et al., 1997; Dieckmann and Doebeli, 1999), in which competing strains coexist and begin to diverge. Understanding how variations in interaction structures and update rules affect evolutionary branching will require formalizing and calculating the establishment probability (Haccou and Iwasa, 1996; Metz et al., 1996; Durinx et al., 2008) - that is, the probability that an invading mutant type will reach a sustainable abundance-for models with nontrivial interaction structure.

\section{Interpretation of structure coefficient}

As is made precise in eq. (7), the structure coefficient quantifies how the outcomes of same-type and different-type interactions affect a mutation's 
fixation probability. Because fixation probability is itself a summary quantity that depends in complex ways on the fundamental events (births, deaths, interactions, etc.) that drive evolution, one may wish to connect the structure coefficient more directly to these fundamental events, and thereby also to other well-studied quantities such as assortment and relatedness.

How to address this challenge depends on the class of models under consideration. Here, as in the work of Tarnita et al. (2009b), evolutionary competition is abstractly represented as a Markov chain. Fixation is represented explicitly, but births, deaths, interactions, and other fundamental events are (in the general case) represented only implicitly, as aspects that may affect transitions between the Markov chain's states, without the dependence of those transitions on those events having to be made explicit or concrete. This abstraction is a strength, in that our results apply to a wide variety of models. However, it also presents a limitation, in that our results cannot be expressed in terms of these fundamental events, because they are represented only implicitly.

This limitation can be overcome by restricting our analyses to a subclass of models for which ecological events are represented more explicitly. An example is the class of models with "global updating" (Nathanson et al., 2009). In this class, the update rule has the property that an individual's reproductive fitness (the number of same-type individuals that an individual leaves after an update; Taylor et al., 2007b; Allen and Tarnita, 2012) is proportional to its expected game payoff. This property can be understood ecologically as requiring that competition to reproduce be global, as local density regulation of survival or fertility otherwise break this proportionality. Notwithstanding this requirement, game interactions may still be local. This class includes most models of well-mixed populations, as well as, for example, Antal et al.'s (2009) model of evolution with phenotypic assortment, Tarnita et al.'s (2009a) model of evolution in social sets, and Allen and Nowak's (2012) model of spatial evolution in cellular populations. For all models in this class, the structure coefficient $\sigma$ can be expressed in terms of the relative frequency of same-type versus different-type game interactions (Nathanson et al., 2009), that is, as a measure of assortment.

For models without global updating, including models in evolutionary graph theory (Ohtsuki et al., 2006; Pacheco et al., 2006b; Taylor et al., 2007a; Allen et al., 2012), the structure coefficient does not merely quantify assortment in game interactions, but also reflects interactions (e.g., competition for space) that are described by the update rule. For example, the Death-Birth 
and Birth-Death update rules on regular graphs (Ohtsuki et al., 2006) yield the same spatial assortment pattern, but result in different values of $\sigma$, due to differences in the way spatial competition is modeled (see Table 1, Ohtsuki et al., 2006, and Nowak et al., 2010b).

Research is ongoing to develop and investigate new classes of IBEG models, with various degrees of generality. For some of these classes, it should be possible to express $\sigma$ in terms of well-studied quantities beyond fixation probabilities. The ultimate result may be a patchwork of classes, with expressions for $\sigma$ that differ across classes but coincide on their intersections.

\section{Discrete versus continuous strategy evolution}

The adaptive dynamics of continuous game strategies in IBEG models differs in several noteworthy ways from discrete strategy evolution. First, for discrete strategies, condition (4) is valid only under weak selection. For continuous strategies, since mutation is incremental in the adaptive dynamics framework, the difference in strategy between competing mutants and residents is assumed to be small. Consequently, fitness differences within the evolving population are small as well. Thus no extra assumption of weak selection is needed for the canonical equation with interaction structure eq. (10) to hold.

Second, condition (4) determines which of the two strategies $A$ and $B$ is more likely to displace the other; however, for discrete strategies, it does not quantify the absolute likelihood of these fixation events. Since absolute fixation probabilities are needed to predict long-term evolutionary dynamics based on strategy substitutions, for discrete strategies, the structure coefficient and effective population size together do not fully characterize the effects of different interaction structures and update rules on long-term evolution. This situation is different for the adaptive dynamics of continuous strategies: the combined assumptions of incremental mutations and of the differentiability of the payoff function $A\left(x^{\prime} ; x\right)$ imply that the first-order behavior of $\rho\left(x^{\prime} ; x\right)$ near $x^{\prime}=x$ is fully determined by $\sigma, N_{\mathrm{e}}$, and the partial derivatives of $A\left(x^{\prime} ; x\right)$ at $x^{\prime}=x$, as we show in Appendix B.

\section{Relation to inclusive fitness theory}

The form of the canonical equation with interaction structure, eq. (10) ,is reminiscent of inclusive fitness theory (Hamilton, 1964a,b; Michod, 1982), in 
that the effects of a trait are partitioned into two terms representing benefits to self and to others, with the second term weighted by a quantity related to interaction patterns.

Yet the canonical equation with interaction structure represents a different perspective than inclusive fitness theory. While no inclusive-fitness analogue of eq. (10) has yet been proposed (see Rousset, 2004, for some steps in this direction), inclusive fitness theory typically focuses on partitioning fitnesses rather than game payoffs (Taylor and Frank, 1996; Taylor et al., 2007b).

While this distinction may at first glance seem immaterial, it does affect the utility and domains of applicability of the two approaches. To apply eq. (10), an IBEG model must be specified - that is, all processes relevant to evolution must be partitioned into those represented by the game, interaction structure, and update rule. This involves an assumption that all outcomes of those processes represented by the game are adequately summarized by the payoffs assigned to the participating individuals. The advantage gained from this assumption is the clean separation of the game from the interaction structure and update rule. These ingredients can then be varied independently in eq. (10), enabling simple yet comprehensive analyses of how the evolutionary trajectories of particular traits vary under different conditions (e.g., Figures 1 and 2).

In contrast, when fitness is partitioned according to inclusive fitness theory, the interaction structure and update rule affect the two fitness terms as well as the relatedness coefficient (Taylor et al., 2007b; Nowak et al., 2010b). These terms must therefore be calculated separately for every model, rendering the aforementioned type of comprehensive analysis more difficult in the inclusive fitness framework.

\section{General approaches to evolutionary theory}

The results presented here apply to a class of evolutionary models, defined by a set of assumptions. Reasoning from general assumptions, rather than specific models, is a relatively new and potentially powerful approach in evolutionary theory. This approach has previously been applied to the dynamics of physiologically structured populations (Metz and Diekmann, 1986; Diekmann et al., 1998, 2001, 2007; Durinx et al., 2008), evolutionary game theory (Tarnita et al., 2009b, 2011), quantitative trait evolution (Champagnat et al., 2006; Simon, 2008), and models with fixed spatial structure (Allen and 
Tarnita, 2012). The advantage of this approach is that general statements, applying to many models or systems, can be proven all at once.

There is another general approach to evolutionary theory, which is termed the "open model" approach by Gardner and West (2006), and is perhaps best typified by the work of Price $(1970,1972)$. The open model approach does not reason from any particular model or set of assumptions. Rather, the starting point is a collection of variables that represent quantities arising in an unspecified evolutionary process. These variables are related to each other using mathematical identities - such as the Price (1970) equationthat hold irrespective of the biological meaning associated to the variables. These identities are then rewritten into forms that can be interpreted as quantitative laws of evolution. For example, versions of Hamilton's rule have been derived through this approach (Queller, 1992; Gardner et al., 2011), as have certain formulations of multilevel selection theory (Price, 1972; Okasha, 2006). The identities derived through this approach are valid in any system for which the variables involved have meaning, but do not, on their own, determine the outcome of any particular model or the consequences of any particular assumption.

A dichotomy has been proposed (Gardner and West, 2006; Gardner et al., 2007, 2011) between "closed model" (modeling in the traditional sense) and open model approaches to evolutionary theory. The approach taken here and by others mentioned above - reasoning from a set of assumptions that delineate a class of models - does not appear to fall under either category. We take the view that there is a spectrum of generality in mathematical approaches to evolutionary theory. At one extreme are detailed models representing specific systems, while the open model approach represents the opposite extreme. Our approach and others like it occupy intermediate positions in this spectrum.

Inherent in the conceptualization of this spectrum is the acknowledgement of a tradeoff between generality of the domain of applicability versus specificity in results. Approaches of intermediate generality (e.g., classical mechanics as derived from Newton's laws of motion) have been tremendously powerful in other fields of science. We believe such approaches represent an exciting growth opportunity for evolutionary theory. 


\section{Limitations}

We caution that, in addition to the assumptions allowing trait evolution to be described by evolutionary game theory, our results depend on the assumptions underlying the adaptive dynamics approach. In particular, our use of condition (4) and other results from evolutionary game theory requires assuming rare and incremental mutations. Relaxation of these assumptions would likely reveal a much more complex interplay between interaction structure, update rule, and game strategy evolution.

Additionally, our results assume that the evolving trait (continuous game strategy) is independent of the interaction structure and update rule. This excludes an important class of models in which behaviors affecting the interaction structure or update rule coevolve with strategy (Le Galliard et al., 2005; Pacheco et al., 2006a,b; Fu et al., 2008; Helbing and Yu, 2009; Perc and Szolnoki, 2010; Wu et al., 2010). For such models, the value of the structure coefficient may vary with the considered game and resident strategy. Establishing the relationship between the structure coefficient and the resident strategy for different games could be an interesting research target in its own right. In such cases, however, the canonical equation with interaction structure, eq. (10), loses the generality that comes from assuming the independence of the game from the interaction structure and update rule, because the aforementioned relationship may be game-specific.

\section{Outlook}

Our results have a number of implications for future research. For one, they suggest extending the focus of analysis from the detailed study of individual models to a general understanding of common features and quantifiable differences between models. Second, the canonical equation with interaction structure, eq. (10), may help illuminate how different interaction structures and update rules affect a wide range of phenomena, such as life-history evolution (Stearns, 1992; Roff, 2002; Crowley and McLetchie, 2002), virulence evolution (Herre, 1993; Boots and Sasaki, 1999; Haraguchi and Sasaki, 2000; Read and Keeling, 2003; Boots et al., 2004), and evolutionary suicide (Ferrière, 2000; Boots and Sasaki, 2002; Gyllenberg et al., 2002; Lion and van Baalen, 2008). Finally, the appearance of the marginal-benefit-to-marginalcost ratio in condition (12) suggests that quantities typically considered in economic theory may be useful for understanding the adaptive dynamics of 
quantitative traits.

\section{Acknowledgements}

B.A. completed part of this research while participating in the Young Scientists Summer Program (YSSP) at the International Institute of Applied Systems Analysis (IIASA) in Laxenburg, Austria. He thanks his YSSP advisor Alexander Tarasiev, as well as Åke Brännström, Brian Fath, Christian Hilbe, J. A. J. Metz, and Corina E. Tarnita for helpful suggestions. Participation in the IIASA Young Scientists Summer Program was made possible by a grant from the National Academy of Sciences Board on International Scientific Organizations, funded by the National Science Foundation under Grant No. OISE-0738129. B.A. is supported by the Foundational Questions in Evolutionary Biology initiative of the John Templeton Foundation. U.D. gratefully acknowledges support by the European Commission, the European Science Foundation, the Austrian Science Fund, the Austrian Ministry for Science and Research, and the Vienna Science and Technology Fund.

\section{A Mathematical framework}

Here we present our mathematical framework and assumptions formally. We state these in the case of a pairwise game, saving the generalization to games with more than two players for Appendix C. Below, we divide our assumptions into those applying to the interaction timescale, the demographic timescale, and the evolutionary timescale. Where needed, explanations in italics provide interpretations of the preceding mathematical statements.

\section{A.1 Interaction timescale: Game strategies and pay- offs}

We assume that the payoff function $A$ satisfies the following condition.

G1. For each $x \in \mathbb{R}^{d}, A$ is positive and twice differentiable in both arguments at $x^{\prime}=x$. 


\section{A.2 Demographic timescale: Resident-mutant compe- tition}

We assume that resident-mutant competition is described by a model satisfying the following conditions.

D1. There is a finite set $S$ with an associated probability distribution $Q$, and a distinguished subset $F_{\mathrm{M}} \subset S$ which is assigned zero probability by $Q$.

$S$ is the set of states. $Q$ is the probability distribution of states corresponding to the appearance of a new mutant strategy. $F_{\mathrm{M}}$ is the set of states corresponding to mutant fixation.

D2. For any payoff matrix

$$
G=\left(\begin{array}{cc}
a_{\mathrm{MM}} & a_{\mathrm{MR}} \\
a_{\mathrm{RM}} & a_{\mathrm{RR}}
\end{array}\right)
$$

there is a collection $\left\{p_{s^{\prime} \mid s}\right\}_{s, s^{\prime} \in S}$ of transition probabilities, giving $S$ the structure of a Markov chain.

D3. The Markov chain associated to any such payoff matrix $G$ has the following properties:

- There is zero probability of transitioning from a state in $F_{\mathrm{M}}$ to a state not in $F_{\mathrm{M}}$.

Mutant fixation is irreversible (barring further mutation).

- For any $s \in S$ that is assigned positive probability by $Q$, and any $s^{\prime} \in F_{\mathrm{M}}$, there is a positive integer $n$ for which the probability of transitioning from $s$ to $s^{\prime}$ in $n$ steps is positive.

Mutant fixation is possible from any initial state.

D4. The transition probabilities $p_{s^{\prime} \mid s}$ vary twice differentiably with respect to the entries of $G$.

D5. If the payoff matrix $G$ is multiplied by a constant $K>0$, the probability $\rho$ that the Markov chain hits $F_{\mathrm{M}}$, given that its initial state is sampled from $Q$, is unaffected.

The evolutionary dynamics are insensitive to the choice of unit by which payoffs are quantified. 
D6. The probability $\rho$ defined in Assumption D5 is increasing in $a_{\mathrm{MM}}$ and $a_{\mathrm{MR}}$, and decreasing in $a_{\mathrm{RM}}$ and $a_{\mathrm{RR}}$, for all values of $a_{\mathrm{MM}}, a_{\mathrm{MR}}, a_{\mathrm{RM}}$, and $a_{\mathrm{RR}}$ sufficiently close to each other (that is, for all values of $a_{\mathrm{MM}}$, $a_{\mathrm{MR}}, a_{\mathrm{RM}}$, and $a_{\mathrm{RR}}$ whose pairwise ratios are sufficiently close to 1 ).

Mutant fixation probability increases with mutant payoffs and decreases with resident payoffs, at least when these payoffs are close to each other.

\section{A.3 Evolutionary timescale: Mutations and trait sub- stitutions}

We place the following assumptions on the adaptive dynamics of game strategies on the evolutionary timescale:

E1. Adaptive evolution follows a Markov jump process with state space $\mathbb{R}^{d}$. This process depends on the following ingredients:

- A payoff function $A(x ; y)$ satisfying Assumption G1,

- A positive real-valued function $u$ on $\mathbb{R}^{d}$,

- A probability measure $U$ on $\mathbb{R}^{d}$, with an associated $\sigma$-algebra of measurable sets,

- A resident-mutant competition model satisfying Assumptions D1D6.

The infinitesimal transition rate to state $x^{\prime} \in \mathbb{R}^{d}$, given that the current state is $x$, is equal to

$$
N u(x) d U\left(x^{\prime}-x\right) \rho\left(x^{\prime} ; x\right) .
$$

Above, $\rho\left(x^{\prime} ; x\right)$ is the fixation probability $\rho$ for the resident-mutant competition model, as defined in Assumption D5, with the entries of $G$ given by

$$
\begin{aligned}
a_{M M} & =A\left(x^{\prime} ; x^{\prime}\right), & a_{M R} & =A\left(x^{\prime} ; x\right), \\
a_{R M} & =A\left(x ; x^{\prime}\right), & a_{R R} & =A(x ; x) .
\end{aligned}
$$

A Markov jump process is a continuous-time Markov process in which the state is constant except for being punctuated by instantaneous jumps to new states. In this case, the state $x \in \mathbb{R}^{d}$ represents the resident strategy, and jumps represent substitution events, through which a new 
mutant strategy displaces the resident strategy. We regard these substitution events as instantaneous from the perspective of long-term evolution. Representing evolution in this way formalizes the assumption, often made in the adaptive dynamics approach, that mutations are rare enough that the resident population can be effectively regarded as typically monomorphic (see, for example, Dieckmann, 1994; Champagnat et al., 2002).

The function $u(x)$ gives the per capita rate at which new mutants appear in a population with resident strategy $x$. The probability measure $U$ represents the distribution of possible mutational steps $x^{\prime}-x$. In the case that $U$ has an associated density function $M$, we can write $d U(z)=$ $M(z) d z$.

Expression (A.1) can be understood by considering that the instantaneous rate at which type $x^{\prime}$ displaces type $x$ is the product of the expected number of mutants born per unit time, $N u(x)$, the (infinitesimal) probability that a mutant is of type $x^{\prime}, d U\left(x^{\prime}-x\right)$, and the probability that mutant type $x^{\prime}$ will displace resident type $x$, should it arise, $\rho\left(x^{\prime} ; x\right)$.

E2. For a random variable $z$ distributed according to $U, \mathrm{E}[z]=0$. Mutation is directionally unbiased.

E3. For a random variable $z$ distributed according to $U, \mathrm{E}\left[|z|^{2}\right] \gg \mathrm{E}\left[|z|^{3}\right]$. The third moment of mutational step size is eclipsed by the second, reflecting an assumption of incremental mutation.

For notational compactness, we additionally assume that $N$ and $U$ do not vary with $x$, and that there is no covariance between the components of a mutational step (that is, $\operatorname{Cov}\left[z_{i}, z_{j}\right]=0$ for all $i, j=1, \ldots, d$ ). These assumptions are inessential and can be relaxed in a straightforward manner, as described by Dieckmann and Law (1996).

Our derivation can also be extended to situations in which the trait space (the set of possible $x$-values) is not the whole of $\mathbb{R}^{d}$, but a domain $D \subset \mathbb{R}^{d}$, as in Examples 1 and 2 of the main text. In this case, the canonical equation, eq. (10), holds at all points $x$ for which the probability that $x+z \notin D$ is negligible. Since the distribution $U$ of mutational steps is presumed to be narrow, this will typically include all points $x$ that are sufficiently far from the boundary of $D$. There are several schemes for extending the canonical 
equation to points near the boundary (Dieckmann et al., 2006). The simplest of these - which we applied in Examples 1 and 2-is to assume that eq. (10) holds in the whole interior of $D$, and that the dynamics on the boundary are given by subtracting, from the vector $d x / d t$ obtained from eq. (10), the component (if any) that points orthogonally outward from $D$.

\section{B Derivation}

We now derive our main result, eq. (10), based on the mathematical framework described above. We first express the expected change in strategy in terms of the fixation probability (Section B.1), and then in terms of game payoffs (Section B.2). These two sections derive the functional form of the canonical equation. Sections B.3 and B.5 relate the coefficients appearing in this equation to the effective population size and structure coefficient, respectively. Sections B.4 and B.6 then establish formulas for, and the positivity of, the effective population size and structure coefficient, respectively.

\section{B.1 Adaptive dynamics in terms of fixation probabili- ties}

The assumption that long-term evolution follows a Markov jump process with infinitesimal transition rates given by eq. (A.1) implies the following equation for the expected change in strategy $x$ per unit time,

$$
\frac{d x}{d t}=N u(x) \int_{\mathbb{R}^{d}} z \rho(x+z ; x) d U(z) .
$$

Since $\rho$ is an absorption probability of a finite Markov chain (Assumptions D1-D3 and D5), and since absorption is possible from any initial state sampled from $Q$ (Assumption D3), $\rho$ varies smoothly with the Markov chain's transition probabilities (see, e.g., Theorem 3.3 of Iosifescu, 1980). Assumption D4 then implies that $\rho$ is twice differentiable with respect to the payoff values $a_{\mathrm{MM}}, a_{\mathrm{MR}}, a_{\mathrm{RM}}$, and $a_{\mathrm{RR}}$. By Assumption G1, $\rho\left(x^{\prime} ; x\right)$ is twice differentiable in $x$ and $x^{\prime}$ at points where $x^{\prime}=x$. We can therefore replace $\rho(x+z ; x)$ by its multivariate Taylor expansion around $z=0$ up to first order,

$$
\rho(x+z ; x)=\rho(x ; x)+\left.\sum_{i=1}^{d} z_{i} \frac{\partial \rho\left(x^{\prime} ; x\right)}{\partial x_{i}^{\prime}}\right|_{x^{\prime}=x}+\mathcal{O}\left(|z|^{2}\right),
$$


yielding

$$
\begin{aligned}
& \frac{d x}{d t}=N u(x) \rho(x ; x) \int_{\mathbb{R}^{d}} z d U(z) \\
&+N u(x) \sum_{i=1}^{d}\left(\left.\frac{\partial \rho\left(x^{\prime} ; x\right)}{\partial x_{i}^{\prime}}\right|_{x^{\prime}=x} \int_{\mathbb{R}^{d}} z z_{i} d U(z)\right) \\
&+\mathcal{O}\left(\int_{\mathbb{R}^{d}}|z|^{3} d U(z)\right)
\end{aligned}
$$

By Assumption E2, the first term on the right-hand side of eq. (B.1) is zero, and by Assumption E3, the third term is negligible relative to the second. Thus eq. (B.1) can be replaced with

$$
\frac{d x}{d t}=N u(x) \sum_{i=1}^{d}\left(\left.\frac{\partial \rho\left(x^{\prime} ; x\right)}{\partial x_{i}^{\prime}}\right|_{x^{\prime}=x} \int_{\mathbb{R}^{d}} z z_{i} d U(z)\right) .
$$

Our assumption of no covariance among the components of $z$ implies that

$$
\int_{\mathbb{R}^{d}} z z_{i} d U(z)=v_{i} e_{i}
$$

where $e_{i}$ is the unit vector for the $i$ th component of $\mathbb{R}^{d}$. The expected change in $x$ is therefore given by the system of equations

$$
\frac{d x_{i}}{d t}=\left.N u(x) v_{i} \frac{\partial \rho\left(x^{\prime} ; x\right)}{\partial x_{i}^{\prime}}\right|_{x^{\prime}=x}
$$

for $i=1, \ldots, d$.

\section{B.2 Adaptive dynamics in terms of game payoffs}

Using the multivariate chain rule, we obtain

$$
\left.\frac{\partial \rho\left(x^{\prime} ; x\right)}{\partial x_{i}^{\prime}}\right|_{x^{\prime}=x}=\left.\left.\sum_{j, k \in\{\mathrm{M}, \mathrm{R}\}} \frac{\partial \rho}{\partial a_{j k}}\right|_{G=\left(\begin{array}{c}
A(x ; x) A(x ; x) \\
A(x ; x) A(x ; x)
\end{array}\right)} \frac{\partial a_{j k}}{\partial x_{i}^{\prime}}\right|_{x^{\prime}=x} .
$$

By Assumption D5, each of the payoff values $a_{j k}$ can be divided by $a_{\mathrm{RR}}=$ $A(x ; x)$ without changing the value of $\rho$. This implies that

$$
\left.\frac{\partial \rho}{\partial a_{j k}}\right|_{G=\left(\begin{array}{cc}
A(x ; x) & A(x ; x) \\
A(x ; x) & A(x ; x)
\end{array}\right)}=\left.\frac{1}{A(x ; x)} \frac{\partial \rho}{\partial a_{j k}}\right|_{G=\left(\begin{array}{cc}
1 & 1 \\
1 & 1
\end{array}\right)},
$$


which, in combination with eq. (B.3), yields

$$
\left.\frac{\partial \rho}{\partial x_{i}^{\prime}}\right|_{x^{\prime}=x}\left(x^{\prime} ; x\right)=\left.\left.\frac{1}{A(x ; x)} \sum_{j, k \in\{\mathrm{M}, \mathrm{R}\}} \frac{\partial \rho}{\partial a_{j k}}\right|_{G=\left(\begin{array}{ll}
1 & 1 \\
1 & 1
\end{array}\right)} \frac{\partial a_{j k}}{\partial x_{i}^{\prime}}\right|_{x^{\prime}=x} .
$$

By the multivariate chain rule, the partial derivatives of the $a_{j k}$ at $x^{\prime}=x$ are given by

$$
\begin{aligned}
& \left.\frac{\partial a_{\mathrm{MM}}}{\partial x_{i}^{\prime}}\right|_{x^{\prime}=x}=\left.\frac{\partial A\left(x^{\prime} ; x\right)}{\partial x_{i}^{\prime}}\right|_{x^{\prime}=x}+\left.\frac{\partial A\left(x ; x^{\prime}\right)}{\partial x_{i}^{\prime}}\right|_{x^{\prime}=x}, \\
& \left.\frac{\partial a_{\mathrm{MR}}}{\partial x_{i}^{\prime}}\right|_{x^{\prime}=x}=\left.\frac{\partial A\left(x^{\prime} ; x\right)}{\partial x_{i}^{\prime}}\right|_{x^{\prime}=x}, \\
& \left.\frac{\partial a_{\mathrm{RM}}}{\partial x_{i}^{\prime}}\right|_{x^{\prime}=x}=\left.\frac{\partial A\left(x ; x^{\prime}\right)}{\partial x_{i}^{\prime}}\right|_{x^{\prime}=x}, \\
& \left.\frac{\partial a_{\mathrm{RR}}}{\partial x_{i}^{\prime}}\right|_{x^{\prime}=x}=0 .
\end{aligned}
$$

We can therefore rewrite eq. (B.4) in the form

$$
\left.\frac{\partial \rho\left(x^{\prime} ; x\right)}{\partial x_{i}^{\prime}}\right|_{x^{\prime}=x}=\frac{\kappa}{A(x ; x)}\left(\left.\frac{\partial A\left(x^{\prime} ; x\right)}{\partial x_{i}^{\prime}}\right|_{x^{\prime}=x}+\left.\kappa^{\prime} \frac{\partial A\left(x ; x^{\prime}\right)}{\partial x_{i}^{\prime}}\right|_{x^{\prime}=x}\right) .
$$

Here, the quantities $\kappa$ and $\kappa^{\prime}$ are given by

$$
\begin{aligned}
\kappa & =\left.\left(\frac{\partial \rho}{\partial a_{\mathrm{MM}}}+\frac{\partial \rho}{\partial a_{\mathrm{MR}}}\right)\right|_{G=\left(\begin{array}{ll}
1 & 1 \\
1 & 1
\end{array}\right)}, \\
\kappa^{\prime} & =\left.\left[\left(\frac{\partial \rho}{\partial a_{\mathrm{MM}}}+\frac{\partial \rho}{\partial a_{\mathrm{RM}}}\right) /\left(\frac{\partial \rho}{\partial a_{\mathrm{MM}}}+\frac{\partial \rho}{\partial a_{\mathrm{MR}}}\right)\right]\right|_{G=\left(\begin{array}{ll}
1 & 1 \\
1 & 1
\end{array}\right)} .
\end{aligned}
$$

We observe that $\kappa$ and $\kappa^{\prime}$ are independent of $A$ and $x$.

Combining eq. (B.5) with eq. (B.2) yields the dynamical equation

$$
\frac{d x_{i}}{d t}=N \frac{u(x) v_{i} \kappa}{A(x ; x)}\left(\left.\frac{\partial A\left(x^{\prime} ; x\right)}{\partial x_{i}^{\prime}}\right|_{x^{\prime}=x}+\left.\kappa^{\prime} \frac{\partial A\left(x ; x^{\prime}\right)}{\partial x_{i}^{\prime}}\right|_{x^{\prime}=x}\right) .
$$

It now only remains to relate $\kappa$ and $\kappa^{\prime}$ to $N_{\mathrm{e}}$ and $\sigma$. We can establish these relationships by considering particularly simple payoff functions $A$ and resident strategies $x$, and substituting these into (B.5). Since $\kappa, \kappa^{\prime}, \sigma$ and $N_{\mathrm{e}}$ are all independent of $A$ and $x$, any relationship derived using a particular choice of $A$ and $x$ will hold generally. 


\section{B.3 Appearance of effective population size}

As motivated in the main text, we define the effective population size for a given resident-mutant competition model as

$$
N_{\mathrm{e}}=\left.\frac{N^{2}}{N-1} \frac{\partial \rho}{\partial s}\right|_{s=0}
$$

where $\rho$ is the fixation probability of a mutant with selective advantage $s$ relative to the resident population.

To relate $N_{\mathrm{e}}$ thus defined to the constant $\kappa$ appearing in (B.7), we must choose a specific payoff function $A$ to substitute into (B.5). As concluded above, a particularly simple choice of $A$ can be made without any loss of generality. We thus consider $A(x ; y)=1+x$, describing frequency-independent selection, so that the link to selective advantage is clear. We choose $x=0$ as the resident strategy. With these choices, mutants and residents have constant payoffs $1+x^{\prime}$ and 1 , respectively (regardless of their interaction partners), so we identify $x^{\prime}$ as the mutant's selective advantage; that is, $x^{\prime}=s$.

Substituting this payoff function $A$ and $x=0$ in eq. (B.5) yields

$$
\left.\frac{\partial \rho\left(x^{\prime} ; 0\right)}{\partial x^{\prime}}\right|_{x^{\prime}=0}=\kappa
$$

Identifying $x^{\prime}$ with $s$ and comparing with eq. (B.8), we obtain

$$
\kappa=N_{\mathrm{e}} \frac{N-1}{N^{2}} .
$$

Since the values of $\kappa$ and $N_{\mathrm{e}}$ do not depend on the game being played, this identity holds for all games.

\section{B.4 Alternate expression for, and positivity of, effec- tive population size}

Equations (B.10) and (B.6) also imply an alternate expression for $N_{\mathrm{e}}$ :

$$
N_{\mathrm{e}}=\left.\frac{N^{2}}{N-1}\left(\frac{\partial \rho}{\partial a_{\mathrm{MM}}}+\frac{\partial \rho}{\partial a_{\mathrm{MR}}}\right)\right|_{G=\left(\begin{array}{ll}
1 & 1 \\
1 & 1
\end{array}\right)},
$$


We can use this expression to show the positivity of $N_{\mathrm{e}}$. Assumption D6 implies that

$$
\left.\frac{\partial \rho}{\partial a_{\mathrm{MM}}}\right|_{G=\left(\begin{array}{ll}
1 & 1 \\
1 & 1
\end{array}\right)}>0,\left.\quad \frac{\partial \rho}{\partial a_{\mathrm{MR}}}\right|_{G=\left(\begin{array}{ll}
1 & 1 \\
1 & 1
\end{array}\right)}>0 .
$$

In combination with eq. (B.11), we can thus conclude that $N_{\mathrm{e}}$ must be positive.

\section{B.5 Appearance of structure coefficient}

To express the constant $\kappa^{\prime}$ appearing in eq. (B.7) in terms of $\sigma$, we first observe that for $x=x^{\prime}$,

$$
G=\left(\begin{array}{ll}
A\left(x^{\prime} ; x^{\prime}\right) & A\left(x^{\prime} ; x^{\prime}\right) \\
A\left(x^{\prime} ; x^{\prime}\right) & A\left(x^{\prime} ; x^{\prime}\right)
\end{array}\right)=A\left(x^{\prime} ; x^{\prime}\right)\left(\begin{array}{cc}
1 & 1 \\
1 & 1
\end{array}\right)
$$

By Assumption D5, the fixation probability $\rho\left(x^{\prime} ; x^{\prime}\right)$ does not depend on the value of $A\left(x^{\prime} ; x^{\prime}\right)$, and is therefore constant with respect to $x^{\prime}$. Differentiating at $x^{\prime}=0$, we obtain

$$
0=\left.\frac{\partial \rho\left(x^{\prime} ; x^{\prime}\right)}{\partial x^{\prime}}\right|_{x^{\prime}=0}=\left.\frac{\partial \rho\left(x^{\prime} ; 0\right)}{\partial x^{\prime}}\right|_{x^{\prime}=0}+\left.\frac{\partial \rho\left(0 ; x^{\prime}\right)}{\partial x^{\prime}}\right|_{x^{\prime}=0}
$$

and therefore

$$
\left.\frac{\partial \rho\left(x^{\prime} ; 0\right)}{\partial x^{\prime}}\right|_{x^{\prime}=0}=-\left.\frac{\partial \rho\left(0 ; x^{\prime}\right)}{\partial x^{\prime}}\right|_{x^{\prime}=0} .
$$

This implies that for all sufficiently small $\left|x^{\prime}\right|$,

$$
\rho\left(x^{\prime} ; 0\right)>\left.\rho\left(0 ; x^{\prime}\right) \Longleftrightarrow \frac{\partial \rho\left(x^{\prime} ; 0\right)}{\partial x^{\prime}}\right|_{x^{\prime}=0}>0 .
$$

As above, we now choose a particular payoff function $A$ to substitute into eq. (B.5). We consider the linear Prisoner's Dilemma with payoff function $A(x ; y)=-c x+b y+1, b>c>0$, and resident and mutant strategies $x=0$ and $x^{\prime}>0$, respectively. The payoff matrix $G$ can then be written as

$$
G=\left(\begin{array}{ll}
1 & 1 \\
1 & 1
\end{array}\right)+x^{\prime}\left(\begin{array}{cc}
b-c & -c \\
b & 0
\end{array}\right)
$$

We observe from the form of this expression that the mutant strategy $x^{\prime}$ can be interpreted as a selection-strength parameter (Nowak et al., 2004; Tarnita 
et al., 2009b). By the defining condition of the structure coefficient, condition 4) in the main text, we obtain, for sufficiently small $x^{\prime}>0$,

$$
\rho\left(x^{\prime} ; 0\right)>\rho\left(0 ; x^{\prime}\right) \Longleftrightarrow \sigma(b-c)-c>b .
$$

Separately, substituting the chosen $A$ and $x=0$ into eq. (B.5) yields

$$
\left.\frac{\partial \rho\left(x^{\prime} ; 0\right)}{\partial x^{\prime}}\right|_{x^{\prime}=0}=\kappa\left(-c+\kappa^{\prime} b\right) .
$$

Applying eq. (B.12) and the positivity of $\kappa$-which follows from eq. (B.10) and the positivity of $N_{\mathrm{e}}$-we obtain

$$
\rho\left(x^{\prime} ; 0\right)>\rho\left(0 ; x^{\prime}\right) \Longleftrightarrow-c+\kappa^{\prime} b>0 .
$$

Comparing condition (B.13) with condition (B.14), we thus see that

$$
\kappa^{\prime}=\frac{\sigma-1}{\sigma+1} .
$$

Again, since the values of $\kappa^{\prime}$ and $\sigma$ do not depend on the game being played, this identity holds for all games.

Substituting eq. (B.10) and eq. (B.15) into eq. (B.7) completes the derivation of the canonical equation of adaptive dynamics with interaction structure, eq. (10).

\section{B.6 Explicit expression for, and positivity of, struc- ture coefficient}

We can also derive an expression for $\sigma$ in terms of partial derivatives of $\rho$ with respect to game payoffs. For this we first observe that, as a consequence of Assumption D5,

$$
\left.\left(\frac{\partial \rho}{\partial a_{\mathrm{MM}}}+\frac{\partial \rho}{\partial a_{\mathrm{MR}}}+\frac{\partial \rho}{\partial a_{\mathrm{RM}}}+\frac{\partial \rho}{\partial a_{\mathrm{RR}}}\right)\right|_{G=\left(\begin{array}{ll}
1 & 1 \\
1 & 1
\end{array}\right)}=0 .
$$

Combining this with eq. (B.15) and eq. (B.6) and solving for $\sigma$ yields

$$
\sigma=\left.\left[\left(\frac{\partial \rho}{\partial a_{\mathrm{MM}}}-\frac{\partial \rho}{\partial a_{\mathrm{RR}}}\right) /\left(\frac{\partial \rho}{\partial a_{\mathrm{MR}}}-\frac{\partial \rho}{\partial a_{\mathrm{RM}}}\right)\right]\right|_{G=\left(\begin{array}{ll}
1 & 1 \\
1 & 1
\end{array}\right)}
$$


We can use this expression to show the positivity of $\sigma$. Assumption D6 implies that

$$
\begin{gathered}
\left.\frac{\partial \rho}{\partial a_{\mathrm{MM}}}\right|_{G=\left(\begin{array}{ll}
1 & 1 \\
1 & 1
\end{array}\right)}>0,\left.\quad \frac{\partial \rho}{\partial a_{\mathrm{MR}}}\right|_{G=\left(\begin{array}{ll}
1 & 1 \\
1 & 1
\end{array}\right)}>0, \\
\left.\frac{\partial \rho}{\partial a_{\mathrm{RM}}}\right|_{G=\left(\begin{array}{ll}
1 & 1 \\
1 & 1
\end{array}\right)}<0,\left.\quad \frac{\partial \rho}{\partial a_{\mathrm{RR}}}\right|_{G=\left(\begin{array}{ll}
1 & 1 \\
1 & 1
\end{array}\right)}<0 .
\end{gathered}
$$

In combination with eq. (B.16), we can thus conclude that $\sigma$ must be positive.

\section{Generalization to multilateral interactions}

The evolutionary dynamics of multilateral interactions, which can be described by games involving three or more players, are often more complex than dynamics arising from pairwise games (Gokhale and Traulsen, 2010). One might therefore expect that the canonical equation, eq. (10), would increase in complexity when applied to $n$-player games for $n \geq 3$. Yet, we show here that-perhaps surprisingly - this is not the case, as long as the game satisfies a reasonable symmetry property.

\section{C.1 Mathematical framework for multilateral interac- tions}

We model a multilateral interaction as an $n$-player continuous game with payoff function $B\left(x ; y_{1}, \ldots, y_{n-1}\right)$, where $x \in \mathbb{R}^{d}$ is the strategy of the focal player receiving the payoff and $y_{1}, \ldots, y_{n-1} \in \mathbb{R}^{d}$ are the strategies of the other participants in the game. We assume $B$ satisfies the following two conditions:

G1. For any $x \in \mathbb{R}^{d}, B$ is positive and twice differentiable in all arguments at $y_{1}=\ldots=y_{n-1}=x$.

G2. The value of $B$ does not change if the arguments $y_{1}, \ldots, y_{n-1}$ are permuted.

We suppose the strategy $x$ evolves according to a model satisfying the analogues of Assumptions D1-D6 and E1-E3 (with the payoff matrix G being replaced by an $n$-dimensional array, as described by Gokhale and Traulsen, 2010). 


\section{C.2 Reduction to pairwise games}

We now show that the adaptive dynamics of $x$ can equivalently be described by a model based on the pairwise game $A$ with $A(x ; y)=B(x ; y, \ldots, y)$. This means that each instance of the game $B$ can effectively be replaced by an interaction in which the game $A$ is played against each interaction partner and the resulting payoffs are averaged. Thus, the adaptive dynamics of $n$ player games reduce to those of pairwise games, as long as the former games are symmetric under the rearrangement of interaction partners (Assumption G2).

This simplification follows from combining the aforementioned symmetry with the multivariate chain rule, as we now demonstrate. By the analogues of eq. (B.2) and eq. (B.4), $d x / d t$ depends on $x$ only insofar as the first-order behavior, in $\left|x^{\prime}-x\right|$, of the entries of the payoff array (the analogue of $G$ ) depends on $x$. By symmetry (Assumption G2), each entry of the payoff array is equal to one of the quantities

$$
\begin{aligned}
& r_{j}=B(x ; \overbrace{x^{\prime}, \ldots, x^{\prime}}^{j}, \overbrace{x, \ldots, x}^{n-j-1}), \\
& m_{j}=B(x^{\prime} ; \overbrace{x^{\prime}, \ldots, x^{\prime}}^{j} \overbrace{x, \ldots, x}^{n-j-1}),
\end{aligned}
$$

for $j=0, \ldots, n-1$. These denote, respectively, the payoff to a resident and to a mutant when interacting with $j$ mutants and $n-j-1$ residents. Expanding $r_{j}$ to first order in $\left|x^{\prime}-x\right|$ yields

$$
\begin{aligned}
r_{j}=B(x ; x, \ldots, x) & \\
& +\left.\left(x^{\prime}-x\right) \frac{\partial B}{\partial x^{\prime}}(x ; \overbrace{x^{\prime}, \ldots, x^{\prime}}^{j}, \overbrace{x, \ldots, x}^{n-j-1})\right|_{x^{\prime}=x}+\mathcal{O}\left(\left|x^{\prime}-x\right|^{2}\right) .
\end{aligned}
$$

Invoking the symmetry property, the multivariate chain rule, and the definition of $A$, we obtain

$$
\begin{aligned}
r_{j} & =B(x ; x, \ldots, x)+\left.j\left(x^{\prime}-x\right) \frac{\partial B}{\partial x^{\prime}}\left(x ; x^{\prime}, x, \ldots, x\right)\right|_{x^{\prime}=x}+\mathcal{O}\left(\left|x^{\prime}-x\right|^{2}\right) \\
& =B(x ; x, \ldots, x)+\left.\frac{j}{n-1}\left(x^{\prime}-x\right) \frac{\partial B}{\partial x^{\prime}}\left(x ; x^{\prime}, \ldots, x^{\prime}\right)\right|_{x^{\prime}=x}+\mathcal{O}\left(\left|x^{\prime}-x\right|^{2}\right) \\
& =A(x ; x)+\left.\frac{j}{n-1}\left(x^{\prime}-x\right) \frac{\partial A\left(x ; x^{\prime}\right)}{\partial x^{\prime}}\right|_{x^{\prime}=x}+\mathcal{O}\left(\left|x^{\prime}-x\right|^{2}\right) .
\end{aligned}
$$


Algebraic rearrangement yields

$$
\begin{aligned}
& r_{j}=\frac{n-j-1}{n-1} A(x ; x) \\
& \quad+\frac{j}{n-1}\left(A(x ; x)+\left.\left(x^{\prime}-x\right) \frac{\partial A\left(x ; x^{\prime}\right)}{\partial x^{\prime}}\right|_{x^{\prime}=x}\right)+\mathcal{O}\left(\left|x^{\prime}-x\right|^{2}\right) .
\end{aligned}
$$

Using the first-order Taylor expansion of $A\left(x ; x^{\prime}\right)$ around $x^{\prime}=x$, we obtain the substitution

$$
A(x ; x)+\left.\left(x^{\prime}-x\right) \frac{\partial A\left(x ; x^{\prime}\right)}{\partial x^{\prime}}\right|_{x^{\prime}=x}=A\left(x ; x^{\prime}\right)+\mathcal{O}\left(\left|x^{\prime}-x\right|^{2}\right),
$$

which finally yields

$$
r_{j}=\frac{n-j-1}{n-1} A(x ; x)+\frac{j}{n-1} A\left(x ; x^{\prime}\right)+\mathcal{O}\left(\left|x^{\prime}-x\right|^{2}\right) .
$$

By an analogous argument, we can show that

$$
m_{j}=\frac{n-j-1}{n-1} A\left(x^{\prime} ; x\right)+\frac{j}{n-1} A\left(x^{\prime} ; x^{\prime}\right)+\mathcal{O}\left(\left|x^{\prime}-x\right|^{2}\right) .
$$

In summary, the payoff obtained from playing the $n$-player game $B$ with a set of $n-1$ interaction partners is equal, to first order in $\left|x^{\prime}-x\right|$, to the arithmetic mean payoff obtained from playing the pairwise game $A$ separately with each interaction partner. Since the canonical equation, eq. (10), depends only on the first derivatives of the payoff function at $x^{\prime}=x$, the adaptive dynamics of $x$ based on the $n$-player game $B$ is therefore fully described by a model based on the pairwise game $A$ with averaged payoffs.

\section{References}

Allen, B., and M. A. Nowak. 2012. Evolutionary shift dynamics on a cycle. Journal of Theoretical Biology 311:28-39.

Allen, B., and C. Tarnita. 2012. Measures of success in a class of evolutionary models with fixed population size and structure. Journal of Mathematical Biology, in press. 
Allen, B., A. Traulsen, C. E. Tarnita, and M. A. Nowak. 2012. How mutation affects evolutionary games on graphs. Journal of Theoretical Biology 299:97-105.

Antal, T., H. Ohtsuki, J. Wakeley, P. D. Taylor, and M. A. Nowak. 2009. Evolution of cooperation by phenotypic similarity. Proceedings of the National Academy of Sciences of the USA 106:8597-8600.

Barton, N. H., and M. Slatkin. 1986. A quasi-equilibrium theory of the distribution of rare alleles in a subdivided population. Heredity 56:409415 .

Boots, M., P. Hudson, and A. Sasaki. 2004. Large shifts in pathogen virulence relate to host population structure. Science 303:842-844.

Boots, M., and A. Sasaki. 1999. 'Small worlds' and the evolution of virulence: infection occurs locally and at a distance. Proceedings of the Royal Society B: Biological Sciences 266:1933-1938.

2002. Parasite-driven extinction in spatially explicit host-parasite systems. American Naturalist 159:706-713.

Brown, J., and T. Vincent. 1987. A theory for the evolutionary game. Theoretical Population Biology 31:140-166.

Campos, P. R. A., and L. M. Wahl. 2009. The effects of population bottlenecks on clonal interference, and the adaptation effective population size. Evolution 63:950-958.

Champagnat, N., R. Ferrière, and G. Ben Arous. 2002. The canonical equation of adaptive dynamics: A mathematical view. Selection 2:73-83.

Champagnat, N., R. Ferrière, and S. Méléard. 2006. Unifying evolutionary dynamics: From individual stochastic processes to macroscopic models. Theoretical Population Biology 69:297-321.

Cressman, R. 1992. The stability concept of evolutionary game theory: A dynamic approach. Springer-Verlag, Berlin.

Crowley, P. H., and D. N. McLetchie. 2002. Trade-offs and spatial life-history strategies in classical metapopulations. American Naturalist 159:190-208. 
Dercole, F., and S. Rinaldi. 2008. Analysis of Evolutionary Processes: The Adaptive Dynamics Approach and its Applications. Princeton University Press, Princeton, NJ.

Dieckmann, U. 1994. Coevolutionary Dynamics of Stochastic Replicator Systems. Reports of the Research Center Jülich. Berichte des Forschungszentrums Jülich (Jül-3018), Jülich, Germany.

Dieckmann, U., and M. Doebeli. 1999. On the origin of species by sympatric speciation. Nature 400:354-357.

Dieckmann, U., M. Heino, and K. Parvinen. 2006. The adaptive dynamics of function-valued traits. Journal of Theoretical Biology 241:370-389.

Dieckmann, U., and R. Law. 1996. The dynamical theory of coevolution: A derivation from stochastic ecological processes. Journal of Mathematical Biology 34:579-612.

Dieckmann, U., R. Law, and J. A. J. Metz, eds. 2000. The Geometry of Ecological Interactions: Simplifying Spatial Complexity. Cambridge University Press, Cambridge.

Dieckmann, U., P. Marrow, and R. Law. 1995. Evolutionary cycling in predator-prey interactions: population dynamics and the Red Queen. Journal of Theoretical Biology 176:91-102.

Diekmann, O., M. Gyllenberg, H. Huang, M. Kirkilionis, J. A. J. Metz, and H. R. Thieme. 2001. On the formulation and analysis of general deterministic structured population models II. Nonlinear theory. Journal of Mathematical Biology 43:157-189.

Diekmann, O., M. Gyllenberg, and J. A. J. Metz. 2007. Physiologically structured population models: Towards a general mathematical theory. Pages 5-20 in Y. Takeuchi, Y. Iwasa, and K. Sato, eds. Mathematics for Ecology and Environmental Sciences, Biological and Medical Physics, Biomedical Engineering. Springer-Verlag, Berlin.

Diekmann, O., M. Gyllenberg, J. A. J. Metz, and H. R. Thieme. 1998. On the formulation and analysis of general deterministic structured population models I. Linear theory. Journal of Mathematical Biology 36:349-388. 
Durinx, M., J. A. J. Metz, and G. Meszéna. 2008. Adaptive dynamics for physiologically structured population models. Journal of Mathematical Biology 56:673-742.

Durrett, R., and S. Levin. 1994. The importance of being discrete (and spatial). Theoretical Population Biology 46:363-394.

Eshel, I., and L. L. Cavalli-Sforza. 1982. Assortment of encounters and evolution of cooperativeness. Proceedings of the National Academy of Sciences of the USA 79:1331-1335.

Ewens, W. J. 1982. On the concept of the effective population size. Theoretical Population Biology 21:373-378.

Fehl, K., D. J. van der Post, and D. Semmann. 2011. Co-evolution of behaviour and social network structure promotes human cooperation. Ecology Letters 14:546-551.

Ferrière, R. 2000. Adaptive responses to environmental threats: evolutionary suicide, insurance, and rescue. Options, Spring 2000:12-16.

Ferrière, R., and J.-F. Le Galliard. 2001. Invasion fitness and adaptive dynamics in spatial population models. Pages 57-79 in J. Clobert, A. Dhondt, E. Danchin, and J. Nichols, eds. Dispersal. Oxford University Press, Oxford.

Fisher, R. A. 1930. The genetical theory of natural selection. Clarendon Press, Oxford.

Fletcher, J. A., and M. Doebeli. 2009. A simple and general explanation for the evolution of altruism. Proceedings of the Royal Society B: Biological Sciences 276:13-19.

Fu, F., C. Hauert, M. A. Nowak, and L. Wang. 2008. Reputation-based partner choice promotes cooperation in social networks. Physical Review E 78:026117.

Fu, F., C. E. Tarnita, N. A. Christakis, L. Wang, D. G. Rand, and M. A. Nowak. 2012. Evolution of in-group favoritism. Scientific Reports 2:460.

Gardner, A. 2010. Sex-biased dispersal of adults mediates the evolution of altruism among juveniles. Journal of Theoretical Biology 262:339-345. 
Gardner, A., and S. A. West. 2006. Demography, altruism, and the benefits of budding. Journal of Evolutionary Biology 19:1707-1716.

Gardner, A., S. A. West, and A. S. Griffin. 2007. Is bacterial persistence a social trait? PLoS ONE 2:e752.

Gardner, A., S. A. West, and G. Wild. 2011. The genetical theory of kin selection. Journal of Evolutionary Biology 24:1020-1043.

Geritz, S. A. H. 2005. Resident-invader dynamics and the coexistence of similar strategies. Journal of Mathematical Biology 50:67-82.

Geritz, S. A. H., M. Gyllenberg, F. J. A. Jacobs, and K. Parvinen. 2002. Invasion dynamics and attractor inheritance. Journal of Mathematical Biology 44:548-560.

Geritz, S. A. H., É. Kisdi, G. Meszéna, and J. A. J. Metz. 1997. Evolutionarily singular strategies and the adaptive growth and branching of the evolutionary tree. Evolutionary Ecology 12:35-57.

Gokhale, C. S., and A. Traulsen. 2010. Evolutionary games in the multiverse. Proceedings of the National Academy of Sciences of the USA 107:55005504.

Grafen, A., and M. Archetti. 2008. Natural selection of altruism in inelastic viscous homogeneous populations. Journal of Theoretical Biology 252:694710 .

Gyllenberg, M., K. Parvinen, and U. Dieckmann. 2002. Evolutionary suicide and evolution of dispersal in structured metapopulations. Journal of Mathematical Biology 45:79-105.

Haccou, P., and Y. Iwasa. 1996. Establishment probability in fluctuating environments: A branching process model. Theoretical Population Biology $50: 254-280$.

Hamilton, W. D. 1964a. The genetical evolution of social behaviour. I. Journal of Theoretical Biology 7:1-16.

. 1964b. The genetical evolution of social behaviour. II. Journal of Theoretical Biology 7:17-52. 
. 1971. Geometry for the selfish herd. Journal of Theoretical Biology 31:295-311.

Hanski, I., and O. E. Gaggiotti. 2004. Ecology, Genetics, and Evolution of Metapopulations. Elsevier, Amsterdam.

Hanski, I., and M. E. Gilpin. 1997. Metapopulation Biology: Ecology, Genetics, and Evolution. Academic Press, San Diego, CA.

Haraguchi, Y., and A. Sasaki. 2000. The evolution of parasite virulence and transmission rate in a spatially structured population. Journal of Theoretical Biology 203:85-96.

Hassell, M. P., H. N. Comins, and R. M. May. 1991. Spatial structure and chaos in insect population dynamics. Nature 353:255-258.

Hauert, C., and M. Doebeli. 2004. Spatial structure often inhibits the evolution of cooperation in the snowdrift game. Nature 428:643-646.

Heino, M., J. A. J. Metz, and V. Kaitala. 1998. The enigma of frequencydependent selection. Trends in Ecology and Evolution 13:367-370.

Helbing, D., and W. Yu. 2009. The outbreak of cooperation among successdriven individuals under noisy conditions. Proceedings of the National Academy of Sciences of the USA 106:3680-3685.

Herre, E. 1993. Population structure and the evolution of virulence in nematode parasites of fig wasps. Science 259:1442-1445.

Hofbauer, J., and K. Sigmund. 1990. Adaptive dynamics and evolutionary stability. Applied Mathematics Letters 3:75-79.

1998. Evolutionary Games and Replicator Dynamics. Cambridge University Press, Cambridge.

2003. Evolutionary game dynamics. Bulletin of the American Mathematical Society 40:479-520.

Ifti, M., T. Killingback, and M. Doebeli. 2004. Effects of neighbourhood size and connectivity on the spatial Continuous Prisoner's Dilemma. Journal of Theoretical Biology 231:97-106. 
Imhof, L. A., and M. A. Nowak. 2006. Evolutionary game dynamics in a Wright-Fisher process. Journal of Mathematical Biology 52:667-681.

2010. Stochastic evolutionary dynamics of direct reciprocity. Proceedings of the Royal Society B: Biological Sciences 277:463-468.

Iosifescu, M. 1980. Finite Markov Processes and Their Applications. Wiley Series in Probability and Mathematical Statistics. Wiley, New York.

Killingback, T., and M. Doebeli. 1996. Spatial evolutionary game theory: Hawks and doves revisited. Proceedings of the Royal Society B: Biological Sciences 263:1135-1144.

. 2002. The continuous prisoner's dilemma and the evolution of cooperation through reciprocal altruism with variable investment. American Naturalist 160:421-438.

Killingback, T., M. Doebeli, and N. Knowlton. 1999. Variable investment, the continuous prisoner's dilemma, and the origin of cooperation. Proceedings of the Royal Society B: Biological Sciences 266:1723-1728.

Kimura, M. 1964. Diffusion models in population genetics. Journal of Applied Probability 1:177-232.

Kimura, M., and G. H. Weiss. 1964. The stepping stone model of population structure and the decrease of genetic correlation with distance. Genetics 49:561-576.

Krakauer, D. C., and M. Pagel. 1995. Spatial structure and the evolution of honest cost-free signalling. Proceedings of the Royal Society B: Biological Sciences 260:365-372.

Le Galliard, J.-F., R. Ferrière, and U. Dieckmann. 2003. The adaptive dynamics of altruism in spatially heterogeneous populations. Evolution 57:117.

2005. Adaptive evolution of social traits: Origin, trajectories, and correlations of altruism and mobility. American Naturalist 165:206-224.

Lessard, S., and V. Ladret. 2007. The probability of fixation of a single mutant in an exchangeable selection model. Journal of Mathematical Biology $54: 721-744$. 
Lieberman, E., C. Hauert, and M. A. Nowak. 2005. Evolutionary dynamics on graphs. Nature 433:312-316.

Lion, S., and M. van Baalen. 2008. Self-structuring in spatial evolutionary ecology. Ecology Letters 11:277-295.

Matsuda, H., N. Ogita, A. Sasaki, and K. Sato. 1992. Statistical mechanics of population. Progress in Theoretical Physics 88:1035-1049.

Maynard Smith, J., and G. R. Price. 1973. The logic of animal conflict. Nature 246:15-18.

McGill, B. J., and J. S. Brown. 2007. Evolutionary game theory and adaptive dynamics of continuous traits. Annual Review of Ecology, Evolution, and Systematics 38:403-435.

Metz, J. A. J. 2011. Effective population sizes and the canonical equation of adaptive dynamics. In Workshop on Stochastic Processes in Cell and Population Biology. Mathematical Biosciences Intitute, Ohio State University.

Metz, J. A. J., and O. Diekmann. 1986. The Dynamics of Physiologically Structured Populations, vol. 68 of Lecture Notes in Biomathematics. Springer-Verlag, Berlin.

Metz, J. A. J., S. A. H. Geritz, G. Meszéna, F. A. Jacobs, and J. S. van Heerwaarden. 1996. Adaptive dynamics: A geometrical study of the consequences of nearly faithful reproduction. Pages 183-231 in S. J. van Strien and S. M. V. Lunel, eds. Stochastic and Spatial Structures of Dynamical Systems. KNAW Verhandelingen, Amsterdam.

Metz, J. A. J., and M. Gyllenberg. 2001. How should we define fitness in structured metapopulation models? Including an application to the calculation of evolutionarily stable dispersal strategies. Proceedings of the Royal Society B: Biological Sciences 268:499-508.

Metz, J. A. J., R. Nisbet, and S. Geritz. 1992. How should we define 'fitness' for general ecological scenarios? Trends in Ecology and Evolution 7:198202. 
Michod, R. E. 1982. The theory of kin selection. Annual Review of Ecology and Systematics 13:23-55.

Mitteldorf, J., and D. S. Wilson. 2000. Population viscosity and the evolution of altruism. Journal of Theoretical Biology 204:481-496.

Moran, P. . A. P. 1958. Random processes in genetics. Mathematical Proceedings of the Cambridge Philosophical Society 54:60-71.

Nakamaru, M., H. Matsuda, and Y. Iwasa. 1997. The evolution of cooperation in a lattice-structured population. Journal of Theoretical Biology 184:65-81.

Nathanson, C. G., C. E. Tarnita, and M. A. Nowak. 2009. Calculating evolutionary dynamics in structured populations. PLoS Computational Biology 5:e1000615.

Nowak, M., and K. Sigmund. 1990. The evolution of stochastic strategies in the prisoner's dilemma. Acta Applicandae Mathematicae 20:247-265.

. 1993. Chaos and the evolution of cooperation. Proceedings of the National Academy of Sciences of the USA 90:5091-5094.

Nowak, M. A. 2006. Five rules for the evolution of cooperation. Science 314:1560-1563.

Nowak, M. A., and R. M. May. 1992. Evolutionary games and spatial chaos. Nature 359:826-829.

Nowak, M. A., A. Sasaki, C. Taylor, and D. Fudenberg. 2004. Emergence of cooperation and evolutionary stability in finite populations. Nature 428:646-650.

Nowak, M. A., and K. Sigmund. 1992. Tit for tat in heterogeneous populations. Nature 355:250-253. 799.

Nowak, M. A., C. E. Tarnita, and T. Antal. 2010a. Evolutionary dynamics in structured populations. Philosophical Transactions of the Royal Society B: Biological Sciences 365:19-30. 
Nowak, M. A., C. E. Tarnita, and E. O. Wilson. 2010b. The evolution of eusociality. Nature 466:1057-1062.

Ohtsuki, H. 2010. Evolutionary games in Wright's island model: Kin selection meets evolutionary game theory. Evolution 64:3344-3353.

Ohtsuki, H., C. Hauert, E. Lieberman, and M. A. Nowak. 2006. A simple rule for the evolution of cooperation on graphs and social networks. Nature 441:502-505.

Ohtsuki, H., and M. A. Nowak. 2008. Evolutionary stability on graphs. Journal of Theoretical Biology 251:698-707.

Ohtsuki, H., M. A. Nowak, and J. M. Pacheco. 2007. Breaking the symmetry between interaction and replacement in evolutionary dynamics on graphs. Physical Review Letters 98:108106.

Okasha, S. 2006. Evolution and the Levels of Selection. Oxford University Press, Oxford.

Pacheco, J. M., A. Traulsen, and M. A. Nowak. 2006a. Active linking in evolutionary games. Journal of Theoretical Biology 243:437-443.

2006b. Coevolution of strategy and structure in complex networks with dynamical linking. Physical Review Letters 97:258103.

Perc, M., and A. Szolnoki. 2010. Coevolutionary games-A mini review. BioSystems 99:109-125.

Price, G. R. 1970. Selection and covariance. Nature 227:520-521.

- 1972. Extension of covariance selection mathematics. Annals of Human Genetics 35:485-490.

Proulx, S. R., and T. Day. 2002. What can invasion analyses tell us about evolution under stochasticity in finite populations? Selection 2:2-15.

Queller, D. 1992. A general model for kin selection. Evolution 46:376-380.

Rand, D. A., H. B. Wilson, and J. M. McGlade. 1994. Dynamics and evolution: Evolutionarily stable attractors, invasion exponents and phenotype dynamics. Philosophical Transactions of the Royal Society B: Biological Sciences 343:261-283. 
Rand, D. G., S. Arbesman, and N. A. Christakis. 2011. Dynamic social networks promote cooperation in experiments with humans. Proceedings of the National Academy of Sciences of the USA 108:19193-19198.

Read, J. M., and M. J. Keeling. 2003. Disease evolution on networks: the role of contact structure. Proceedings of the Royal Society B: Biological Sciences 270:699-708.

Roca, C. P., J. A. Cuesta, and A. Sánchez. 2009. Effect of spatial structure on the evolution of cooperation. Physical Review E 80:046106.

Roff, D. A. 2002. Life History Evolution. Sinauer Associates, Sunderland, MA.

Rousset, F. 2004. Genetic Structure and Selection in Subdivided Populations. Princeton University Press, Princeton, NJ.

Santos, F. C., and J. M. Pacheco. 2005. Scale-free networks provide a unifying framework for the emergence of cooperation. Physical Review Letters 95:98104.

Simon, B. 2008. A stochastic model of evolutionary dynamics with deterministic large-population asymptotics. Journal of Theoretical Biology 254:719730 .

Stearns, S. C. 1992. The Evolution of Life Histories. Oxford University Press, Oxford.

Szabó, G., and G. Fáth. 2007. Evolutionary games on graphs. Physics Reports 446:97-216.

Tarnita, C. E., T. Antal, H. Ohtsuki, and M. A. Nowak. 2009a. Evolutionary dynamics in set structured populations. Proceedings of the National Academy of Sciences of the USA 106:8601-8604.

Tarnita, C. E., H. Ohtsuki, T. Antal, F. Fu, and M. A. Nowak. 2009b. Strategy selection in structured populations. Journal of Theoretical Biology 259:570-581.

Tarnita, C. E., N. Wage, and M. A. Nowak. 2011. Multiple strategies in structured populations. Proceedings of the National Academy of Sciences of the USA 108:2334-2337. 
Taylor, P. D. 1992. Altruism in viscous populations-An inclusive fitness model. Evolutionary Ecology 6:352-356.

Taylor, P. D., T. Day, and G. Wild. $2007 a$.

Evolution of cooperation in a finite homogeneous graph. Nature 447:469472.

$2007 b$. From inclusive fitness to fixation probability in homogeneous structured populations. Journal of Theoretical Biology 249:101-110.

Taylor, P. D., and S. A. Frank. 1996. How to make a kin selection model. Journal of Theoretical Biology 180:27-37.

Taylor, P. D., and L. B. Jonker. 1978. Evolutionary stable strategies and game dynamics. Mathematical Biosciences 40:145-156.

Tilman, D., and P. M. Kareiva. 1997. Spatial Ecology: The Role of Space in Population Dynamics and Interspecific Interactions. Princeton University Press, Princeton, NJ.

Traulsen, A., and M. A. Nowak. 2006. Evolution of cooperation by multilevel selection. Proceedings of the National Academy of Sciences of the USA 103:10952-10955.

Traulsen, A., J. M. Pacheco, and M. A. Nowak. 2007. Pairwise comparison and selection temperature in evolutionary game dynamics. Journal of Theoretical Biology 246:522-529.

van Baalen, M. 2002. Contact networks and the evolution of virulence. Pages 85-103 in U. Dieckmann, J. A. J. Metz, M. W. Sabelis, and K. Sigmund, eds. Adaptive Dynamics of Infectious Diseases: In Pursuit of Virulence Management. Cambridge University Press, Cambridge.

van Baalen, M., and D. A. Rand. 1998. The unit of selection in viscous populations and the evolution of altruism. Journal of Theoretical Biology 193:631-648.

van Veelen, M., J. García, D. G. Rand, and M. A. Nowak. 2012. Direct reciprocity in structured populations. Proceedings of the National Academy of Sciences of the USA 109:9929-9934. 
Wahl, L. M., and M. A. Nowak. 1999a. The continuous prisoner's dilemma II. Linear reactive strategies with noise. Journal of Theoretical Biology 200:323-338.

1999b. The continuous prisoner's dilemma I. Linear reactive strategies. Journal of Theoretical Biology 200:307-321.

Weibull, J. W. 1997. Evolutionary Game Theory. MIT Press.

Werfel, J., and Y. Bar-Yam. 2004. The evolution of reproductive restraint through social communication. Proceedings of the National Academy of Sciences of the USA 101:11019-11024.

Wilson, D. S. 1977. Structured demes and the evolution of groupadvantageous traits. American Naturalist 111:157-185.

Wilson, D. S., G. Pollock, and L. Dugatkin. 1992. Can altruism evolve in purely viscous populations? Evolutionary Ecology 6:331-341.

Wright, S. 1931. Evolution in Mendelian populations. Genetics 16:97-159. 1943. Isolation by distance. Genetics 28:114-138.

Wu, B., D. Zhou, F. Fu, Q. Luo, L. Wang, A. Traulsen, and O. Sporns. 2010. Evolution of cooperation on stochastic dynamical networks. PLoS ONE 5:1560-1563. 
Table 1: Effective Population Size and Structure Coefficient for IBEG Models

\begin{tabular}{|c|c|c|}
\hline Model & Effective pop. size, $N_{\mathrm{e}}$ & Structure coefficient, $\sigma$ \\
\hline $\begin{array}{l}\text { Well-mixed Wright-Fisher } \\
\text { process (Fisher, 1930; } \\
\text { Wright, 1931) }\end{array}$ & $N$ & $\frac{N-2}{N}$ \\
\hline $\begin{array}{l}\text { Well-mixed continuous-time } \\
\text { birth-death process } \\
\text { (Dieckmann, 1994) }\end{array}$ & $\frac{N}{2}$ & $\frac{N-2}{N}$ \\
\hline $\begin{array}{l}\text { Well-mixed Moran process } \\
\text { (Nowak et al., 2004) }\end{array}$ & $\frac{N}{2}$ & $\frac{N-2}{N}$ \\
\hline $\begin{array}{l}\text { Transitive graph, } \\
\text { birth-death updating } \\
\text { (Ohtsuki et al., 2006; Taylor } \\
\text { et al., 2007a) }\end{array}$ & $\frac{N}{2}$ & $\frac{N-2}{N}$ \\
\hline $\begin{array}{l}\text { Transitive graph, } \\
\text { death-birth updating } \\
\text { (Ohtsuki et al., 2006; Taylor } \\
\text { et al., 2007a) }\end{array}$ & N 0 & $\frac{(k+1) N-4 k}{(k-1) N}$ \\
\hline $\begin{array}{l}\text { Group selection without } \\
\text { migration (Traulsen and } \\
\text { Nowak, 2006) }\end{array}$ & $\frac{N}{2} \frac{\ell+n_{\mathrm{g}}-2}{N-1}$ & $1+\frac{2 n_{\mathrm{g}}-4}{\ell}$ \\
\hline $\begin{array}{l}\text { Group selection with } \\
\text { migration (Traulsen and } \\
\text { Nowak, 2006) }\end{array}$ & $\frac{N}{2(N-1)}\left(\ell-1+\frac{\ell(q-\lambda)\left(n_{\mathrm{g}}-1\right)}{\lambda+\ell q}\right)$ & $1-\frac{2}{\ell}+\frac{2 q\left(n_{\mathrm{g}}-1\right)}{\lambda+\ell q}$ \\
\hline $\begin{array}{l}\text { Infinite island model } \\
\text { (Wright, 1931; Taylor, 1992; } \\
\text { Ohtsuki, 2010) }\end{array}$ & Unknown & 1 \\
\hline $\begin{array}{l}\text { Phenotypic assortment } \\
\text { (Antal et al., 2009) }\end{array}$ & Unknown & $\frac{1+4 N \nu}{2+4 N \nu}\left(1+\sqrt{\frac{3+12 N \nu}{3+4 N \nu}}\right)$ \\
\hline
\end{tabular}

$N=$ population size, $k=$ degree of graph, $n_{\mathrm{g}}=$ number of groups, $\ell=$ number of individuals per group, $q=$ group splitting probability, $\lambda=$ migration rate, $\nu=$ phenotypic mutation rate. Transitive graphs are spatially homogeneous; see Taylor et al. (2007a) and Grafen and Archetti (2008). The result for the island model of Wright (1931) and Taylor (1992) applies to the limit of infinitely many islands, as analyzed by Ohtsuki (2010). 


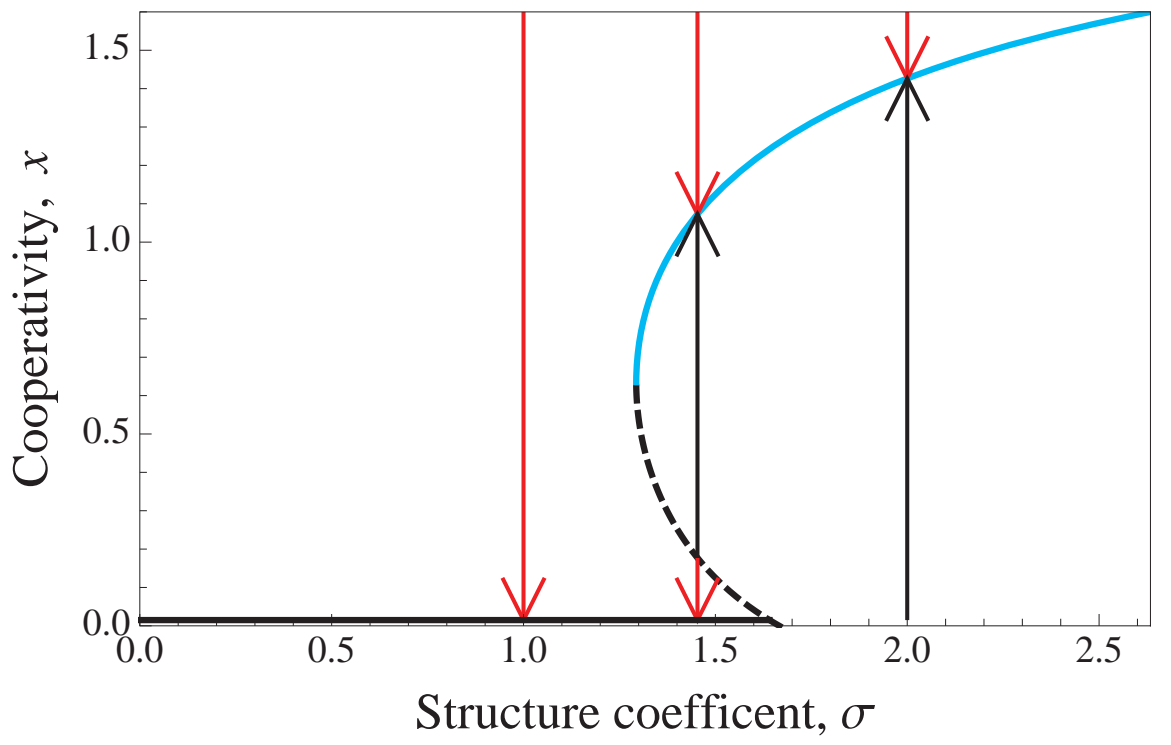

Figure 1: Bifurcation diagram showing the adaptive dynamics of cooperativity in eq. (11) under different interaction structures and update rules. The structure coefficient $\sigma$ serves as a control parameter. Arrows indicate the direction of cooperativity evolution. For this example, $B(x)=$ $4 x+4 x^{2}$ and $C(x)=x+x^{5} / 5$. Equilibrium points lie on the curve $\sigma=\left[B^{\prime}(x)+C^{\prime}(x)\right] /\left[B^{\prime}(x)-C^{\prime}(x)\right]$ and on the line $x=0$. At $\sigma \approx 1.29$ a saddle-node bifurcation occurs, resulting in the creation of a stable equilibrium with positive cooperativity and an unstable cooperativity threshold. As $\sigma$ increases, the cooperativity at the stable equilibrium grows and its basin of attraction expands. The leftmost arrow at $\sigma=1$ corresponds to a large well-mixed population. The central arrows at $\sigma=109 / 75 \approx 1.45$ could correspond, for example, to a $5 \times 5$ square lattice with death-birth updating, or to 19 groups of size 75 without migration. The rightmost arrows at $\sigma=2$ could correspond to a large cyclically structured population, or 12 groups of size 10 without migration. See Table 1 for these and other interpretations of values of $\sigma$ in terms of specific IBEG models. 


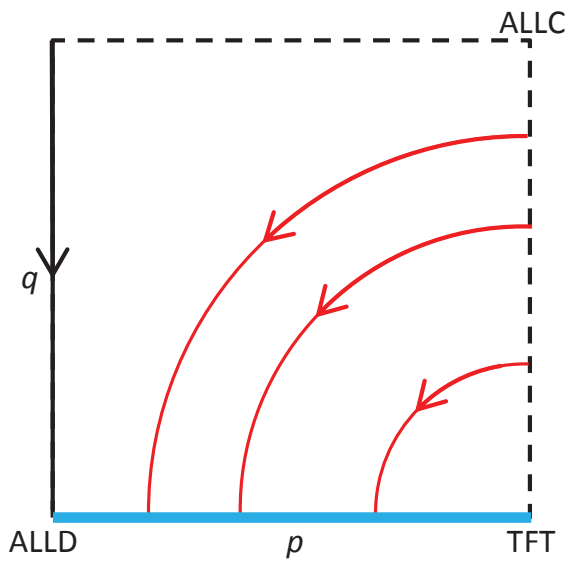

(a) $\sigma \rightarrow 0$

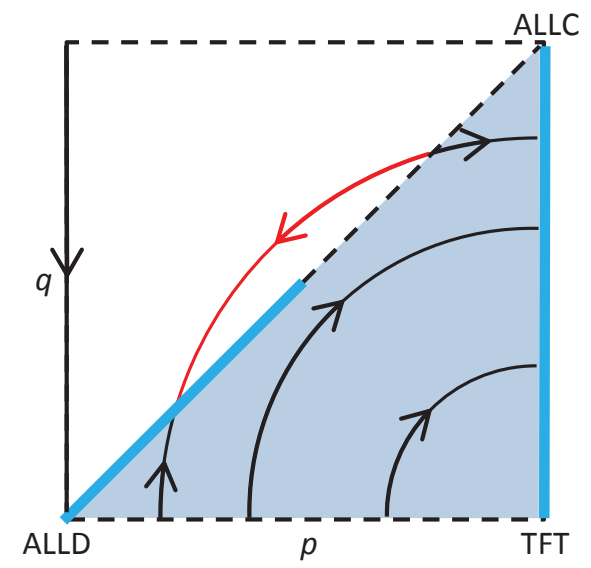

(c) $\sigma=(b+c) /(b-c)$

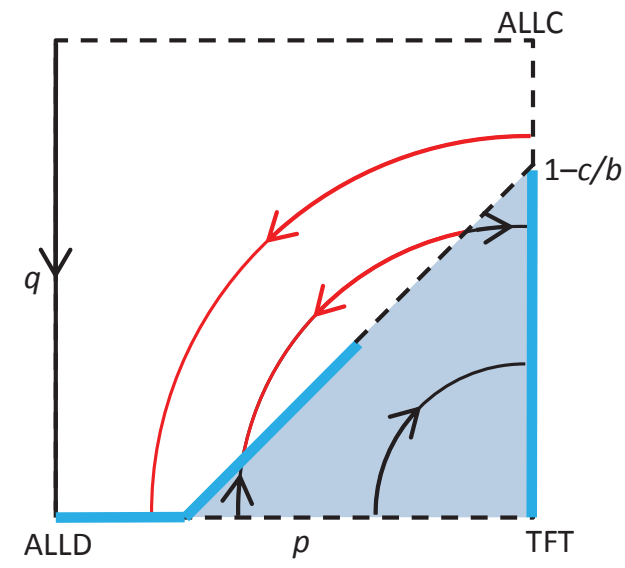

(b) $\sigma=1$

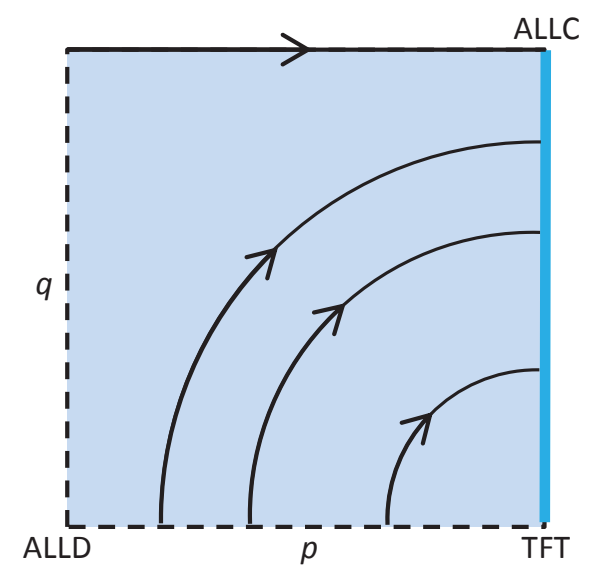

(d) $\sigma \rightarrow \infty$

Figure 2: Adaptive dynamics of stochastic reactive strategies in the iterated Prisoner's Dilemma for different interaction structures and update rules. The value of the structure coefficient $\sigma$ increases from panel (a) to panel (d). Thin continuous lines depict evolutionary trajectories, with arrowheads and line color indicating the direction of evolutionary change. Thick continuous lines, rendered in blue/gray, depict stable equilibria, while thin dashed lines depict unstable equilibria or boundary points. The evolutionary dynamics along the boundaries are obtained by ignoring the outward-pointing component, if any, of $d x / d t$. (a) In the limit $\sigma \rightarrow 0$, all equilibrium strategies deterministically respond to $D$ with $D$. In this case, TFT is the only equilibrium strategy that achieves a positive payoff against itself. (b-d) As $\sigma$ increases, a range of "generous tit-for-tat" (GTFT; Nowak and Sigmund, 1990) strategies become stable equilibria. These GTFT strategies are characterized by $p=1$ and $q>0$, and are therefore situated on the right-hand edge of the domain. Analysis of condition (15) shows that the GTFT strategy $(1, q)$ is a stable equilibrium if and only if $q<[2 \sigma(-c+b)] /[\sigma(-c+b)+c+b]$. (b) For $\sigma=1$, corresponding to large well-nßBxed populations, the classical GTFT strategy $(1,1-c / b)$ (Nowak and Sigmund, 1990) becomes stable. (c) For $\sigma=(b+c) /(b-c)$ ALLC is a stable equilibrium, along with the full range of GTFT strategies. (d) In the limit $\sigma \rightarrow \infty$, the set of GTFT strategies is a global evolutionary attractor. 